\title{
Neural Processing of Acoustic and Electric Interaural Time Differences in Normal-Hearing Gerbils
}

\author{
는 Maike Vollmer \\ Comprehensive Hearing Center, University Hospital Wuerzburg, 97080 Wuerzburg, Germany, Department of Otolaryngology-Head and Neck Surgery, \\ University Hospital Magdeburg, Otto von Guericke University, 39120 Magdeburg, Germany, and Center for Learning and Memory Research, Leibniz \\ Institute for Neurobiology, 39118 Magdeburg, Germany
}

Bilateral cochlear implants (CIs) provide benefits for speech perception in noise and directional hearing, but users typically show poor sensitivity to interaural time differences (ITDs). Possible explanations for this deficit are deafness-induced degradations in neural ITD sensitivity, between-ear mismatches in electrode positions or activation sites, or differences in binaural brain circuits activated by electric versus acoustic stimulation. To identify potential limitations of electric ITD coding in the normal-hearing system, responses of single neurons in the dorsal nucleus of the lateral lemniscus and in the inferior colliculus to ITDs of electric (biphasic pulses) and acoustic (noise, clicks, chirps, and tones) stimuli were recorded in normal-hearing gerbils of either sex. To maintain acoustic sensitivity, electric stimuli were delivered to the round window. ITD tuning metrics (e.g., best ITD) and ITD discrimination thresholds for electric versus transient acoustic stimuli (clicks, chirps) obtained from the same neurons were not significantly correlated. Across populations of neurons with similar characteristic frequencies, however, ITD tuning metrics and ITD discrimination thresholds were similar for electric and acoustic stimuli and largely independent of the spectrotemporal properties of the acoustic stimuli when measured in the central range of ITDs. The similarity of acoustic and electric ITD coding on the population level in animals with normal hearing experience suggests that poorer ITD sensitivity in bilateral CI users compared with normalhearing listeners is likely due to deprivation-induced changes in neural ITD coding rather than to differences in the binaural brain circuits involved in the processing of electric and acoustic ITDs.

Key words: binaural hearing; cochlear implant; inferior colliculus; interaural time difference; lateral lemniscus; temporal processing

\section{Significance Statement}

Small differences in the arrival time of sound at the two ears (interaural time differences, ITDs) provide important cues for speech understanding in noise and directional hearing. Deaf subjects with bilateral cochlear implants obtain only little benefit from ITDs. It is unclear whether these limitations are due to between-ear mismatches in activation sites, differences in binaural brain circuits activated by electric versus acoustic stimulation, or deafness-induced degradations in neural ITD processing. This study is the first to directly compare electric and acoustic ITD coding in neurons of known characteristic frequencies. In animals with normal hearing, populations of auditory brainstem and midbrain neurons demonstrate general similarities in electric and acoustic ITD coding, suggesting similar underlying central auditory processing mechanisms.

\section{Introduction}

In normal-hearing listeners, interaural time differences (ITDs) are the dominant cue for localizing sounds containing low fre-

\footnotetext{
Received Nov. 22, 2017; revised June 4, 2018; accepted June 4, 2018

Author contributions: M.V. wrote the first draft of the paper; M.V. edited the paper; M.V. designed research; M.V. performed research; M.V. analyzed data; M.V. wrote the paper.

This work was supported by the Deutsche Forschungsgemeinschaft (Priority Program 1608 "Ultrafast and Temporally Precise Information Processing: Normal and Dysfunctional Hearing", Grants V0 640/2-1 and V0 640/2-2 to M.V.) and the Interdisciplinary Center for Clinical Research (Grant N-100 to M.V.). I thank Otto Gleich and Peter Heil for valuable comments on the manuscript, Armin Wiegner and Tristan Bremer for programming and hardware support, Martin Kempe for help with ABR recordings, Barbara Kellner for assistance with histological preparations, and Rainer Brandtner for laboratory animal care. Cochlear implants were provided by MedEl (Innsbruck, Austria).

The author declares no competing financial interests.
}

quencies in azimuth (Wightman and Kistler, 1992; Macpherson and Middlebrooks, 2002). In bilateral cochlear implant (CI) users, ITD discrimination is typically poorer than in normalhearing listeners and highly variable across subjects. The range of stimulus conditions (e.g., stimulus rates) over which ITD sensitivity is observed is restricted in bilateral CI users and, even under optimal conditions (i.e., direct stimulation through a single in-

Correspondence should be addressed to Maike Vollmer, Department of Otolaryngology-Head and Neck Surgery University Hospital Magdeburg, Otto von Guericke University, Leipziger Strasse 44, 39120 Magdeburg, Germany. E-mail: maike.vollmer@med.ovgu.de.

DOI:10.1523/JNEUROSCI.3328-17.2018

Copyright $\odot 2018$ the authors $\quad 0270-6474 / 18 / 386949-18 \$ 15.00 / 0$ 
teraural electrode pair), only few CI users achieve ITD sensitivity similar to that in normal-hearing listeners (for review, Kan and Litovsky, 2015; Laback et al., 2015). Benefits from bilateral CIs compared with monaural CIs are mainly based on attending to the ear with the better signal-to-noise ratio (Litovsky et al., 2006; Ricketts et al., 2006) and on using interaural level differences (Seeber and Fastl, 2008; Aronoff et al., 2010; van Hoesel, 2012; ILDs). One possible explanation for poorer ITD discrimination in bilateral CI users compared with normal-hearing listeners are deafness-induced degradations in neural ITD sensitivity (for studies in cats, see Tillein et al., 2010 and Hancock et al., 2010, 2013; for a study in humans, see Litovsky et al., 2010). Other possible explanations are between-ear mismatches in electrode positions or activation sites of auditory nerve fibers (ANFs) (He et al., 2012; Hu and Dietz, 2015; Joshi et al., 2017) or differences in the binaural brain circuits activated by electric versus acoustic stimulation.

Studies in bilaterally implanted adult cats with short deafness durations (7-14 d) showed that a high proportion of neurons in the inferior colliculus (IC) was sensitive to ITDs of electric pulse trains. However, some ITD tuning metrics of these neurons for electric stimuli [best ITD, ITD at maximum slope (ITDms)] differed significantly from those obtained for acoustic broadband noise in normal-hearing cats (Smith and Delgutte, 2007). The characteristic frequencies (CFs) of neurons in the deafened animals could not be determined, and it remained unclear whether differences in the CF or the short deafness durations in the electrically stimulated animals were responsible for the observed discrepancies between electric and acoustic ITD tuning. Moreover, although general similarities in neural ITD sensitivity in the IC to acoustic broadband and narrowband stimuli have been reported (Yin et al., 1986; Carney and Yin, 1989; Plauška et al., 2017), different spectrotemporal acoustic stimulus properties likely influence neural ITD coding.

To identify intrinsic differences between electric and acoustic ITD coding, basic ITD tuning features and neural ITD discrimination thresholds to electric stimulation of the round window (biphasic pulses) and to acoustic stimulation with sounds of different spectral (narrowband tones versus broadband noise, clicks, and chirps) and temporal properties (sustained tones and noise versus transient clicks and chirps) were characterized for the central range of ITDs in overlapping populations of predominantly low-frequency neurons in normal-hearing gerbils.

Human studies have used trains of brief, bandlimited acoustic pulses to mimic the spectrotemporal properties of pulsatile electric stimuli (McKay and Carlyon, 1999; Majdak et al., 2006; Kan et al., 2013). If similar mechanisms are involved in the binaural processing of transient stimuli of either mode, then responses to acoustic and electric stimuli should display similar ITD characteristics. In the present study, this hypothesis was tested by comparing ITD processing of electric pulses with that of acoustic clicks and chirps in the same neurons.

Acoustic studies have shown that ITD response properties of neurons in the dorsal nucleus of the lateral lemniscus (DNLL) and the IC are similar (Kuwada et al., 2006). Electric and acoustic stimulation might engage different binaural excitatory and inhibitory brain circuits that may affect ITD processing of electric stimuli in DNLL and IC differently. Therefore, neural responses to ITDs of electric and acoustic stimuli were compared in the two nuclei.

On the population level, the basic nature of neural ITD processing in normal-hearing animals was similar for both stimulus modes and largely independent of the spectrotemporal proper- ties of the acoustic stimuli. However, for a given neuron, acoustic ITD coding did not predict electric ITD coding. The characteristic features of electric and acoustic ITD processing were similar in DNLL and IC.

\section{Materials and Methods}

\section{Surgical procedures}

All experiments were performed in accordance with the German animal welfare law (54-2531.01-13/07, 55.2-2531.01-37/08, and 55.2-2531.0142/13). Adult Mongolian gerbils (Meriones unguiculatus) of either sex were bilaterally implanted with round window electrodes at the time of the experiment. All surgical procedures and electrophysiological recordings were conducted under general anesthesia. Animals were initially sedated by intraperitoneal injection [7.5 $\mu \mathrm{l} / \mathrm{g}$ bodyweight (BW)] using a mixture of ketamine $(7.5 \mathrm{mg} / \mathrm{ml})$, xylazine $(1.3 \mathrm{mg} / \mathrm{ml})$, glucose $(16.1$ $\mathrm{mg} / \mathrm{ml})$, and $\mathrm{NaCl}(48.4 \mathrm{mg} / \mathrm{ml})$. Anesthesia was maintained at an areflexic level using a continuous subcutaneous infusion via a syringe pump $(3.2 \mu \mathrm{l} / \mathrm{g} \mathrm{BW} / \mathrm{h})$. Body temperature was maintained at $\sim 37^{\circ} \mathrm{C}$ using a feedback-controlled heating blanket (BC4; Thomas Wulf Elektronik). Dexamethasone (3 $\mu \mathrm{g} / \mathrm{g} \mathrm{BW} / 6 \mathrm{~h})$ and glycopyrrolate $(0.011 \mu \mathrm{g} / \mathrm{g}$ $\mathrm{BW} / 12 \mathrm{~h}$ ) were administered subcutaneously to prevent brain edema and to reduce salivation, respectively. Respiration rate and reflexes were monitored regularly during the experiment.

Before implantation of the round window electrodes, normal acoustic sensitivity was confirmed by the presence of acoustically evoked auditory brainstem responses (aABRs) to clicks at $\leq 40 \mathrm{~dB}$ peak sound pressure level (pSPL) (for details, see "ABRs" section). For implantation, the skin was incised along the midline of the skull and postauricularly on both sides. A metal headpost was fixed to the cranium using a bone screw $(1.6 \times 2.4 \mathrm{~mm}$; Plastics One) and dental hybrid composite (Charisma; Heraeus). On both sides, the tympanic bulla was opened, and a custommade electrode with a single ball-shaped contact (diameter $\sim 0.3 \mathrm{~mm}$; $\mathrm{MedEl}$ ) was inserted into the round window niche and secured in place using cyanoacrylate tissue adhesive (Vetbond; 3M Animal Care Products). A reference electrode was placed in the neck muscle behind each bulla. The round window approach offered two important advantages for the present study: First, acoustic sensitivity in the implanted ears was maintained and thus allowed for both acoustic and electric stimulation of the cochlea. Second, monopolar round window stimulation activates neurons across the entire tonotopic axis, including low-frequency ITDsensitive neurons (Leake et al., 1995). Successful implantation was confirmed by electrically evoked ABRs (eABRs) to electric pulses (for details, see "ABRs" section).

The animal was then mounted in a modified stereotaxic frame (David Kopf Instruments). A craniotomy $\left(\sim 2.5 \times 2 \mathrm{~mm}^{2}\right)$ was performed $\sim 1$ $\mathrm{mm}$ left of the midline of the skull and just caudal to bregma. The craniotomy was extended caudally to the rostral aspect of the transverse sinus, and the dura was removed.

After the electrophysiological recordings, animals were killed by an overdose of anesthesia and transcardially perfused with a buffered $\mathrm{NaCl}$ solution, followed by a $4 \%$ paraformaldehyde solution. After $2 \mathrm{~d}$ in sucrose solution, the brain was frozen at $-20^{\circ} \mathrm{C}$ and processed histologically.

\section{Stimuli, stimulus generation, and calibration}

For acoustic stimulation, pure tones $\left(50 \mathrm{~ms}, 5 \mathrm{~ms} \cos ^{2}\right.$ rise/fall time), broadband unfiltered white noise ( $100 \mathrm{~ms}, 5 \mathrm{~ms} \cos ^{2}$ rise/fall time), trains of monophasic square-wave unfiltered condensation clicks (100 $\mu \mathrm{s}$ ), and trains of upward frequency-modulated sweeps ("chirps") were used. The frequency range of the chirp was narrower than that tested by Earl and Chertoff (2012), but the rate of the frequency change was identical. The frequency of the chirp was swept from $160 \mathrm{~Hz}$ to $20 \mathrm{kHz}$ in 3.23 $\mathrm{ms}$ to compensate for the cochlear traveling wave delay and to achieve simultaneous activation along the gerbil's basilar membrane (for details, see Earl and Chertoff, 2012). For electric stimulation, biphasic squarewave pulses ( $80 \mu \mathrm{s} /$ phase, $10 \mu \mathrm{s}$ interphase interval, cathodic leading) were used.

Acoustic and electric stimuli were digitally generated by a real-time processor (RX8; Tucker-Davies Technologies; $100 \mathrm{k} \mathrm{samples/s).} \mathrm{Acoustic}$ stimuli were passed through a programmable attenuator (PA5; Tucker- 
A

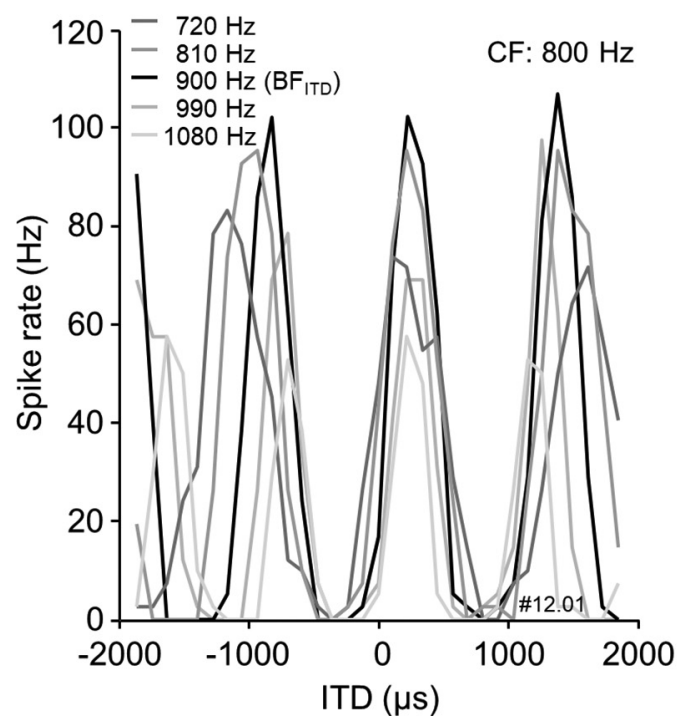

B

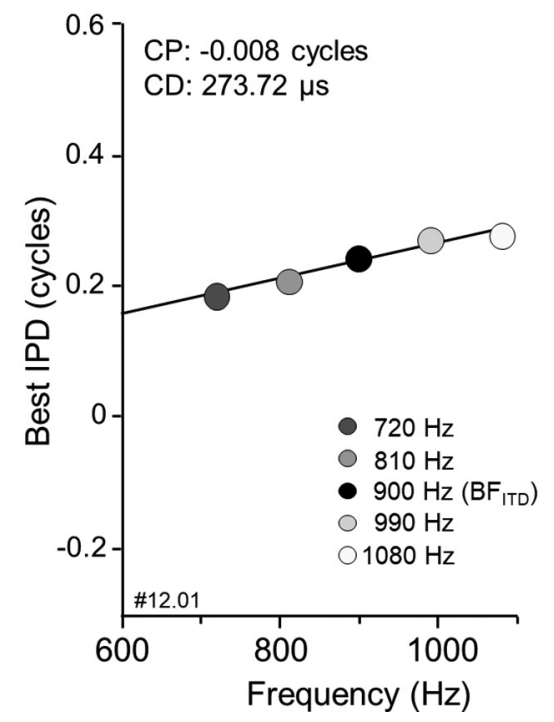

Figure 1. Responses of a single peak-type neuron to ITDs of pure tones. $A$, ITD functions in response to tones of five different frequencies. The spike rate is maximally modulated at a $B F_{\text {ITD }}$ of 900 $\mathrm{Hz}$ (black). $\boldsymbol{B}$, Corresponding best IPDs were calculated via vector analysis and are plotted against tone frequency to calculate the $\mathrm{CP}$ and $\mathrm{CD}$.

Davies Technologies), amplified (AMP84; Thomas Wulf Elektronik) and delivered by closed-field speakers (Eartone $5 \mathrm{~A} 50 \Omega$; Etymotic) that were sealed to the external meati. Before each recording experiment, acoustic clicks were calibrated to dB pSPL (Burkard, 2006) using a custom-made $20 \mu \mathrm{l}$ coupler (silicone tube) and a probe microphone (4182; Brüel \& Kjær). The acoustic output of the speaker was amplified (Nexus 2690; Brüel \& Kjær) and digitized (200 k samples/s, RX6). Tone stimuli were calibrated in a frequency-specific manner to $\mathrm{dB}$ SPL using white noise $(2 \mathrm{~s})$. Its spectrum was obtained by fast Fourier transformation and corrected for the known frequency response of the probe microphone. Electric stimuli were passed through a multichannel attenuator (ATT20; Thomas Wulf Elektronik) and delivered to the round window electrode through a custom-made, optically isolated current source (ICS5; Thomas Wulf Elektronik). Electric stimulation levels in $\mathrm{dB}$ were calibrated relative to $100 \mu \mathrm{A}$ peak to peak $\left(\mu \mathrm{A}_{\mathrm{pp}}\right)$.

\section{$A B R s$}

All recordings were performed in a double-walled, sound-attenuated, and electrically shielded chamber (Industrial Acoustics). To assess neural sensitivity to both acoustic and electric stimulation of the cochleae, aABRs and eABRs were measured monaurally to low rate $(21 \mathrm{~Hz})$ acoustic clicks and electric pulses, respectively (for details see "Stimuli, stimulus generation, and calibration" section; Wiegner et al., 2016). For eABR measures, electric pulses alternated in polarity to minimize the stimulus artifact. Subcutaneous electrodes were placed in the ipsilateral neck (active), the snout (reference), and the back (ground) of the animal. ABRs were recorded using Tucker-Davies Technologies System 3 hardware and software (BioSigRP: $21 \mathrm{~Hz}, 500-1500$ repetitions). Stimuli were amplified (RA4LI), digitized (RA4PA; $25 \mathrm{k} \mathrm{samples/s),} \mathrm{and} \mathrm{filtered} \mathrm{(RX5:} 300-$ $3000 \mathrm{~Hz}, 50 \mathrm{~Hz}$ notch filter). The ABR threshold was defined as the lowest stimulation level that evoked a reproducible response according to visual criteria. Before and after implantation, all animals included in this study showed normal sensitivity to acoustic clicks (threshold criterion $\leq 40 \mathrm{~dB}$ pSPL). Differences in eABR thresholds between the two ears were $\leq 3 \mathrm{~dB}$.

\section{Single neuron recordings}

Single neuron responses were recorded extracellularly using tungsten microelectrodes (impedance 4-7 M $\Omega$; Microprobe). The recording electrode was tilted from medial to lateral by $5^{\circ}$ and gradually advanced from dorsomedial to ventrolateral into the IC and DNLL using a remotely controlled piezoelectric microdrive (PM 101; Märzhäuser) held in a modified micromanipulator (David Kopf Instruments). Recordings were amplified (RA16AC; Tucker-Davies Technologies), digitized (25 k samples/s, RA16PA; Tucker-Davies Technologies), filtered (RX5; Tucker-Davies Technologies; 600-7000 Hz), and stored on a PC. Isolation of action potentials (spikes) from single neurons was verified by shape stability and offline spike cluster analysis (Brainware; TuckerDavies Technologies; see Fig. 2B).

Typically, either $100 \mathrm{~ms}$ noise bursts or low-rate electric pulses [2-10 pulses per second (pps)] were delivered monaurally and binaurally and used as search stimuli. Once a single neuron was isolated, 50 or $100 \mathrm{~ms}$ pure tones were presented monaurally and diotically (zero ITD and zero ILD) at randomized frequency-level combinations ranging from 0.2 to $20 \mathrm{kHz}$ (2-5 steps/octave) and from 0 to $80 \mathrm{~dB}$ SPL ( $10 \mathrm{~dB}$ steps) to determine the neuron's frequency-level response area and CF (i.e., the tone frequency that elicited responses at the lowest sound level). To characterize the neuron's ITD response type (for details, see "ITD tuning" section), ITD sensitivity to pure tones $(50 \mathrm{~ms})$ was assessed by presenting a matrix of stimulus frequencies and ITDs at $\sim 20 \mathrm{~dB}$ above the diotic threshold at CF. Five frequencies centered around the frequency that elicited the maximum modulation in spike rate across ITDs [best ITD frequency $\left(\mathrm{BF}_{\mathrm{ITD}}\right)$; see Fig. $1 A$ ] were then presented over a range of ITDs that was equivalent to at least two cycles of the stimulus frequency and 10 interaural phase differences (IPDs) per cycle (Pecka et al., 2008). If necessary, the stimulus level was adjusted to increase spike rate modulation to different ITDs.

Rate-level functions for monaural and diotic stimulation with single electric pulses ( 1 or $2 \mathrm{~dB}$ steps) were used to determine the electric dynamic range (level range for the $10-90 \%$ range of driven spike rate) and response threshold (the level at $10 \%$ of driven spike rate). Rate-level functions for acoustic clicks, chirps, and noise were derived using sound levels between 0 and $80 \mathrm{~dB}(\mathrm{p}) \mathrm{SPL}$ in steps of 5 or $10 \mathrm{~dB}$ that were presented in random order.

To allow for stimulus-locked responses and to avoid onset-only responses of IC and DNLL neurons to repetitive stimuli, ITD sensitivity for transient stimuli (electric pulses, acoustic clicks, and chirps) was typically tested with low-rate (20 pps) periodic stimulus trains of $300 \mathrm{~ms}$ duration, presented every $600 \mathrm{~ms}$. Electric pulse trains were presented at 1-4 dB above the diotic threshold for single pulses, and acoustic click and chirp trains were usually presented at $20 \mathrm{~dB}$ above the diotic threshold for single stimuli. If necessary, stimulus level and stimulus rate (typically between 10 and 40 pps) were adjusted to increase ITD sensitivity. ITD 
sensitivity for noise was tested using single $100 \mathrm{~ms}$ noise bursts presented every $350-400 \mathrm{~ms}$ at $\sim 10-20 \mathrm{~dB}$ above the diotic threshold.

ITDs for broadband stimuli (electric pulses, acoustic clicks, chirps, and noise) typically covered ranges from \pm 1500 to $\pm 3000 \mu$ s with step sizes of 100-200 $\mu$ s. Positive ITD values refer to contralateral leading stimuli and negative ITDs to ipsilateral leading stimuli. In low-CF neurons, ITD functions for acoustic stimuli and for electrophonic responses to electric stimuli can show multiple peaks that occur at intervals that are approximately equal to 1/CF (Yin et al., 1986, 1987; Carney and Yin, 1989; see Figs. $2 E, 3 A, B, D, F, H)$. Because of time constraints in singleunit recordings, the ITD ranges for broadband stimuli did not always cover two full cycles of the CF. In the case of narrowly tuned neurons, the ITD range was reduced to as low as $\pm 200 \mu$ s with step sizes of $10-25 \mu \mathrm{s}$ to improve the resolution. ITDs were always presented in random order. Typically, each stimulus was repeated 20 times.

Responses of single neurons were usually not available for all stimulus types because neurons were lost before recordings were complete or because neurons did not respond to all stimuli. Moreover, in initial experiments, a neuron was not always tested for ITD sensitivity to broadband stimuli if it was not ITD sensitive to tones. This resulted in a bias toward low-CF neurons ( $\leq 2 \mathrm{kHz}$; see "CF" section in Results). In later experiments, the sequence of recordings was flexible and, if time permitted, all neurons were tested for ITD sensitivity to broadband stimuli. This increased the number of high-CF neurons $(>2 \mathrm{kHz})$.

\section{Electrophonic versus electroneural responses}

Electric stimulation of the hearing cochlea can evoke both electrophonic responses that closely resemble responses to acoustic stimulation and electroneural responses that result from direct electric activation of the auditory nerve. Compared with electrophonic responses, electroneural responses are characterized by higher thresholds, shorter latencies, higher synchronization to the electric stimulus, and narrower dynamic ranges (Moxon, 1971; Hartmann et al., 1984; Lusted and Simmons, 1984; van den Honert and Stypulkowski, 1984; Miller et al., 2006; Vollmer et al., 2008; Sato et al., 2016).

To ensure that the reported data were restricted to those obtained with electroneural activation, responses to electric pulses were evaluated over large ranges of stimulus current. For the neurons included, electric response thresholds were usually $>200 \mu \mathrm{A}_{\mathrm{pp}}$, and the responses displayed narrow dynamic ranges (typically between 2 and $6 \mathrm{~dB}$ ). Response thresholds and dynamic ranges were similar to those for electroneural responses recorded in a different cohort of deafened gerbils (Wiegner et al., 2016). Moreover, some of the animals included in this study were deafened after collecting the data for this study, and subsequent responses to electric stimulation of the deaf cochleae were recorded. Within-animal comparisons confirmed the similarity in response patterns, thresholds, and dynamic ranges before and after deafening. Across the entire dynamic range, single-neuron responses to electric stimulation were strongly synchronized and displayed no abrupt changes in latency with increasing stimulus current. Again, these observations argue for electroneural responses.

Poststimulus time histograms (PSTHs) constructed from the responses of low-CF ANFs to clicks show multiple peaks occurring at intervals of $\sim 1 / \mathrm{CF}$ (Kiang et al., 1965; Pfeiffer and Kim, 1972; Carney and Yin, 1988; Guinan et al., 2005; for review, see Heil and Peterson, 2015). The PSTHs of ANFs to electric pulses can also show such multiple peaks if the responses are of electrophonic origin. These peaks should be reflected as multiple peaks in the ITD functions of DNLL and IC neurons. However, in the present study, the ITD functions for electric stimuli did not display multiple peaks even when recorded over a range of ITDs sufficiently wide to include 2/CF (see Figs. 2E, F, 3A-C,E, $G, 4 C$ ). Also, none of the PSTHs of IC neurons to single monaural and diotic electric pulses had multiple peaks at intervals of $1 / \mathrm{CF}$ (data not shown). These response patterns support direct electroneural rather than electrophonic activation.

In addition, within the narrow dynamic ranges typical for direct electroneural responses, the currents used to obtain ITD functions were usually set close to saturation. These relatively high currents and the use of monopolar (round window) stimulation further reduce the probabil- ity of electrophonic responses to electric pulses (Moxon, 1971; Lusted and Simmons, 1984; van den Honert and Stypulkowski, 1984). The response patterns of the reported neurons to electric stimulation were consistent with direct electroneural activation.

\section{Data analysis}

ITD sensitivity. Spike rates were plotted against ITD to obtain ITD functions (see Figs. 1, 2, 3,4). To characterize ITD sensitivity independently of the shape of the ITD function, an ANOVA calculation derived from the raw spike counts for each ITD and each stimulus trial (Hancock et al., 2010) was performed. Onset responses were included in the spike count calculation. For each neuron, the ratio of the variance of the mean response attributable to changes in ITD to the total variance of the response across all repetitions and ITDs was determined (ITD signal to total variance ratio, STVR). STVR values range from 0 (when the mean response is the same for all ITDs) to 1 (when a stimulus with a given ITD elicits the same number of spikes on every presentation, when this is true for all ITDs, and when the mean number of spikes is not the same for all ITDs). Neural responses were defined as ITD sensitive and analyzed further if there were significant differences in the mean number of spikes across the ITDs tested ( $F$ test, $p<0.025$; Hancock et al., 2010).

ITD tuning. For ITD-sensitive neurons, the shapes of ITD functions in response to electric pulses and acoustic clicks, chirps, and noise were categorized using four fitting functions (peak, trough, biphasic, and sigmoid; criterion: $R^{2} \geq 0.314$; Smith and Delgutte, 2007; see Fig. 4). Each ITD function was assigned to the category that provided the best fit (i.e., the smallest sum of squared errors). The fitting functions of peak shape and biphasic ITD functions possess three parameters that characterize ITD tuning: best ITD (ITD at maximum spike rate), half-width as a measure of sharpness of tuning (ITD range at half-height of the Gaussian fit for peak shape functions and of the positive-going component for biphasic functions; Hancock et al., 2013), and ITDms (ITD at the steepest slope where the spike rate is most sensitive to changes in ITD; for peakshape ITD functions, ITDms was determined from the slope closest to zero ITD).

ITD functions for clicks, chirps, and noise can have multiple peaks (see Figs. 2E, $F, 3 A, B, D, F, H)$. Multipeak ITD functions that could not be fitted unambiguously (criterion: $R^{2} \leq 0.314$ ) were excluded from shape categorization, and ITD tuning metrics were not determined (see Fig. $3 D, F$ ). If the ITD functions displayed a single dominant peak (or trough), then the best fit was used for shape categorization (see Fig. $3 H$ ).

ITD functions for pure tones typically have multiple peaks (see Fig. $1 A$ ) and were categorized as peak type, trough type, or intermediate type based on their characteristic phase (CP, see below). For each stimulus frequency, the best IPD and vector strength were analyzed using a vector analysis (Goldberg and Brown, 1969; Yin and Kuwada, 1983a). Best IPD (criterion $p<0.001$; Raleigh test; Mardia, 1972) was plotted against stimulus frequency. A nonweighted least-squares linear regression was calculated if at least three IPD values were available (see Fig. $1 B$ ). The linearity of the IPD versus frequency relationship was assessed using the mean-square-error evaluation described by Yin and Kuwada (1983b). Using this method, $91 \%(126 / 139)$ of the neurons displayed linearity. This proportion is similar to the $93 \%$ reported by Siveke et al. (2006), who used a weighted mean-square-error measure (Kuwada et al., 1987). The CP is defined as the $y$-intercept of the regression line at $0 \mathrm{~Hz}$ and describes the mean interaural phase at which the characteristic delay (CD) occurs; that is, where the ITD functions for different frequencies display constant relative amplitude (Yin and Kuwada, 1983b; Kuwada et al., 1987). CP values at or near 0 cycles (between 0 and \pm 0.18 cycles) indicate CDs at or close to the peaks of the ITD functions (peak-type neurons). In contrast, $\mathrm{CP}$ values near 0.5 cycles (within \pm 0.17 of \pm 0.5 cycles) indicate CDs at or close to the troughs of the ITD functions (trough-type neurons). Intermediate-type neurons are characterized by CP values between 0.18 and 0.33 and between -0.18 and -0.33 cycles. Thus, unlike ITD functions for electric pulses and acoustic clicks, chirps, and noise (see Fig. 4), the ITD functions for tones were not categorized based on the shape fit and were, therefore, not included in the quantitative analysis of response shapes shown in Figure $5 \mathrm{~A}$. However, to compare tuning metrics quantitatively across all stimuli, best ITD, half- 
width, and ITDms for tones were calculated based on the peak fit (positive Gaussian function; Smith and Delgutte, 2007) to the most central peak of the tone ITD function at $\mathrm{BF}_{\mathrm{ITD}}$ (Fig. $4 A$, central peak data are from the neuron in Fig. $1 A$ ). Because ITD tuning metrics in response to tones largely depend on stimulus frequency, the following analyses conservatively focus only on peak-type neurons. These neurons typically display the maximal responses to tones (i.e., best ITD) at the same ITD or at similar ITDs across frequencies (Yin and Chan, 1990; Batra et al., 1997; Joris and Yin, 1998).

Neural discrimination thresholds. A modified version of the standard separation $D$, originally proposed by Sakitt (1973), was used to estimate ITD discrimination of single neurons. The modified measure is analogous to $d^{\prime}$ used in psychophysical studies to quantify discrimination performance and is given by the following equation:

$$
D_{I T D, I T D+\Delta I T D}=\frac{\left|\mu_{I T D}-\mu_{I T D+\Delta I T D}\right|}{\sqrt{\left(\sigma_{I T D}^{2}+\sigma_{I T D+\Delta I T D}^{2}\right) / 2}},
$$

where $\mu_{\mathrm{ITD}}$ and $\mu_{\mathrm{ITD}+\Delta \mathrm{ITD}}$ are the means of the spike counts at two different ITDs, and $\sigma_{\text {ITD }}$ and $\sigma_{\text {ITD }+\Delta \text { ITD }}$ are their corresponding SDs. The geometric mean of variances in the original definition of standard separation $D$ (Sakitt, 1973) was replaced by the arithmetic mean to avoid invalid equations in cases where the spike count variance is zero for one of the ITDs (Smith and Delgutte, 2007). The neural discrimination threshold (NDT) is defined as the difference in ITD relative to a reference ITD that is required for the standard separation $D$ to reach a value of one. NDT was computed in both positive and negative directions relative to a reference of 0 ITD. Only the lowest of these two NDTs is reported in Figure 11.

When recorded from the same neurons, responses to acoustic clicks and chirps were similar for any of the measures extracted (i.e., ITD tuning metrics, NDTs; Wilcoxon signed-rank test, all $p>0.18$ ). Nevertheless, data obtained in response to clicks and chirps will be reported separately in the following population analyses and will only be pooled for within-neuron comparisons between electric pulses and acoustic clicks or chirps.

\section{Recording sites and histology}

In each animal, we determined the locations of the recording sites by stereotaxic coordinates relative to lambda. To identify the recording sites and to assign them to either IC or DNLL, 2-3 electrolytic lesions $(8 \mu \mathrm{A}$, 2.5-3 $\mathrm{min}$ ) were made while the electrode was withdrawn.

Brains were sectioned in coronal orientation (25-50 $\mu \mathrm{m}$ thickness) and stained with Berliner Blau and Nissl using a standard protocol. Using light microscopy, the lesion sites and/or the track of the recording electrode were photographed, and the recording locations were determined based on the coordinates.

In some cases, the locations of DNLL and IC could also be identified using physiological criteria. When advanced ventrally, the electrode typically encountered a silent zone (width $\sim 500 \mu \mathrm{m}$ ) between the ventral part of the IC and the dorsal part of the DNLL, where no neurons could be isolated. The following entry into the DNLL was marked by an abrupt increase in spontaneous activity and neuronal discharge rates (Kuwada et al., 2006).

\section{Experimental design and statistical analysis}

The study compares ITD characteristics (best ITD, half-width, ITDms, NTD) of single neurons in response to five stimulus types (electric pulses, acoustic clicks, chirps, noise, and tones) (for details, see "Database" section in Results).

The data were tested for normality and equal variance (SigmaPlot 13.0). If these conditions were not met, the nonparametric KruskalWallis (K-W) test was used, followed by Dunn's test for pairwise multiple comparisons. Results are reported as medians \pm quartile deviation (Q). If the samples were partially dependent and the data were sufficiently normal, a one-way ANOVA was used with neurons as random effects [restricted maximum likelihood (REML) estimates of the variances; JMP 13.2], followed by Bonferroni-adjusted multiple $t$ tests with random effects. In the case of skewed data (half-width and NDT), the measures were log-transformed to approach normality. For the compar- ison of neuronal responses from DNLL and IC, the Mann-Whitney rank-sum test was used. For matched-pairs comparisons of neural responses to acoustic clicks or chirps and electric pulses, the Wilcoxon signed-rank test was used. For categorial data distributions, the $\chi^{2}$ test or the two-sample Kolmogorov-Smirnov test (K-S) was used.

\section{Results}

\section{Database}

This report includes recordings from 183 single neurons in 48 normal-hearing gerbils. The number of neurons per animal varied between 1 and 11, with an average and SD of $3.8 \pm 2.9$. Seventy-one $(39 \%)$ of these neurons were located in the IC, 83 (45\%) in the DNLL, and $29(16 \%)$ could not unambiguously be assigned to either IC or DNLL because anatomical (electrolytic lesions) or physiological identification was not available. For each stimulus type, at least $74 \%$ of the recorded ITD functions exhibited significant ITD sensitivity.

\section{Shapes of ITD functions and ITD response types}

Figure $2 A$ shows responses of an example IC neuron to low-rate (20 pps) trains of electric pulses (left), acoustic clicks (middle), and acoustic chirps (right). ITDs varied between $\pm 1900 \mu \mathrm{s}$. For all three stimulus types, the neuron's responses were locked to the stimuli and clearly varied with the ITD, with a preference for positive ITDs (contralateral leading, i.e., corresponding to locations in the contralateral hemifield). Stable spike waveforms and isolation of response clusters in the feature space (Fig. 2B) revealed that the responses to the different stimuli were obtained from the same neuron. Figure $2 C$ displays the corresponding ITD functions for the three stimulus types. All three functions nearly overlap, are peak shaped, and narrowly tuned with a best ITD around $+220 \mu \mathrm{s}$. Nonetheless, when recorded from the same neuron, ITD functions for transient stimuli of different modes can clearly differ. Figure $2 D-F$, shows ITD functions for electric pulses and acoustic clicks from three other neurons. These functions display different ITD tuning widths and best ITDs for the different stimulus modes. Moreover, in low-CF neurons, ITD functions for acoustic stimuli can have multiple peaks that occur at intervals of $\sim 1 / \mathrm{CF}$ (Yin et al., 1986, 1987; Carney and Yin, 1989; Fig. 2E; see also Fig. $3 A, B, D, F, H$ ). Multiple peaks were not observed in ITD functions for electric pulses, consistent with the notion that the responses were evoked by electroneural rather than electrophonic activation (Fig. 2C-F; see also Figs. $3 A-$ $C, E, G, 4 C, D)$. A multipeak ITD function for clicks was observed in one high-CF neuron (Fig. $2 F$ ). In this case, however, the peaks did not occur at intervals of $1 / \mathrm{CF}$, so the underlying mechanisms are likely not related to cochlear frequency selectivity.

For the broadband stimuli, the shapes of the ITD functions of ITD sensitive neurons in normal-hearing animals were assigned to one of four categories (peak, trough, sigmoid, or biphasic) that were previously used to categorize data from bilaterally deafened and implanted cats (Smith and Delgutte, 2007; Hancock et al., 2010, 2013). Figure 4 shows examples of the four shape categories. Each ITD function was assigned to the category that provided the best fit (see Materials and Methods). Because the distributions of shape categories of DNLL and IC neurons did not differ for any of the stimuli tested $(\mathrm{K}-\mathrm{S}$, all $p>0.05)$, shape categories were pooled across all neurons for further analysis. Figure $5 A$ shows the fraction of each shape category for electric pulses, acoustic clicks, chirps, and noise. Peak-shape ITD functions were the most common independent of the stimulus mode (electric/acoustic) or the temporal properties (transient or sustained) of the broadband acoustic stimuli. 

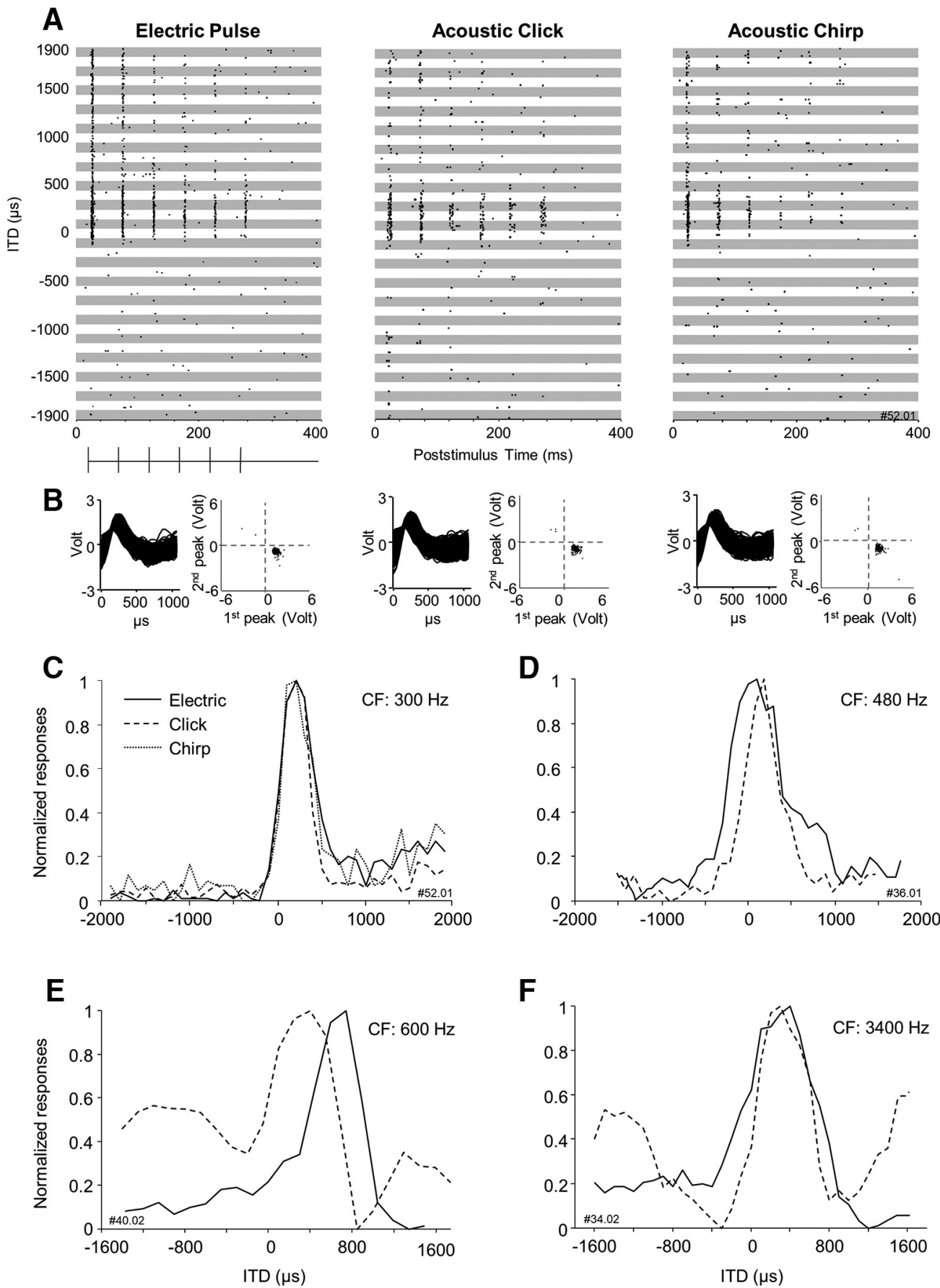

Figure 2. Responses of single neurons to ITDs of transient electric and acoustic stimuli. $\boldsymbol{A}$, Raster plots showing responses of a single neuron to trains of electric pulses (left), acoustic clicks (middle), and chirps (right) presented at different ITDs (gray and white bars). The stimulus train is identical for all three stimuli ( 6 stimuli, $20 \mathrm{pps}$ ) and is shown below the plot for electric pulses. $\boldsymbol{B}$, Spike waveforms and feature spaces for the same responses as in $A$. Isolation of spikes from single neurons was verified by the stability of the spike shape and offline feature space analysis for spike clusters. C, Peak-shape ITD functions of the same neuron as in $A$ in response to electric pulses, acoustic clicks, and chirps. To compare the shapes of the ITD functions, each function was normalized to its minimum and maximum. In this example, the ITD functions for all three stimulus types and both stimulus modes are peak shaped and similar. $\boldsymbol{D}-\boldsymbol{F}$, ITD functions of three other neurons for electric pulses and acoustic clicks. Functions for both stimulus modes differ with respect to tuning width and/or best ITD. ITD functions for acoustic clicks can display multiple peaks that are not observed for electric pulses $(\boldsymbol{E}, \boldsymbol{F})$.

For tone stimulation, the ITD response types (not the shapes) of neurons are typically categorized based on a vector analysis (Fig. 1; Yin and Kuwada, 1983a; see Materials and Methods). ITD response types to tones are often thought to be associated with specific neural mechanisms underlying ITD sensitivity. Peaktype neurons are associated with the coincidence of binaural excitatory inputs in the medial superior olive (MSO), whereas trough-type neurons are thought to reflect ipsilateral excitation and contralateral inhibition, as found in the lateral superior olive (LSO) (Yin and Chan, 1990; Batra et al., 1997; Joris and Yin, 1998). Neurons with ITD functions deviating from peak or trough type may receive inputs from different brainstem nuclei or inputs from several MSO neurons with different best ITDs (McAlpine et al., 1998; Siveke et al., 2006). However, some stud- 


\section{Electric pulses vs. Noise}

A

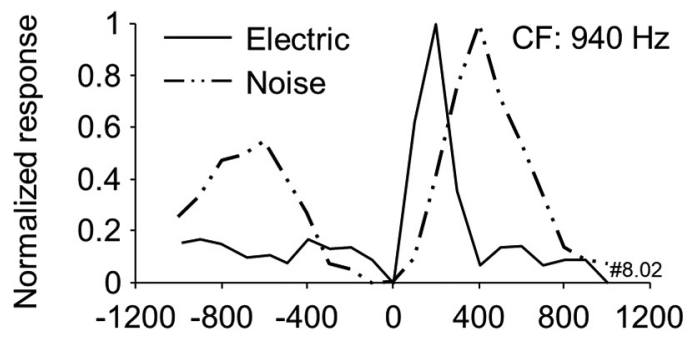

Electric

\section{C}

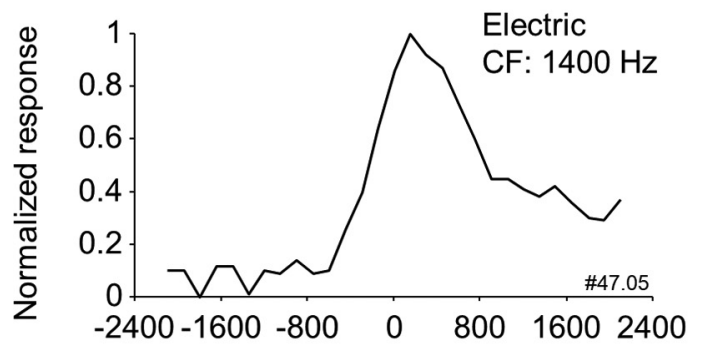

E

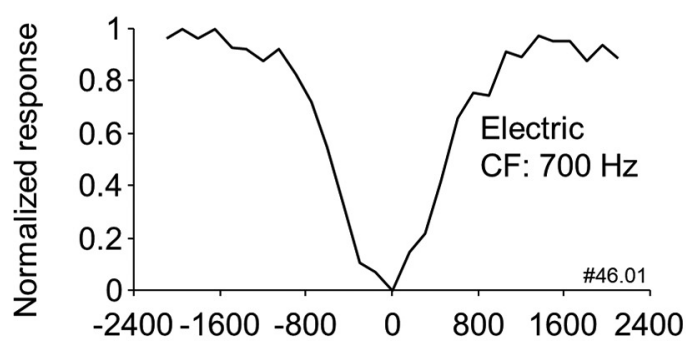

G

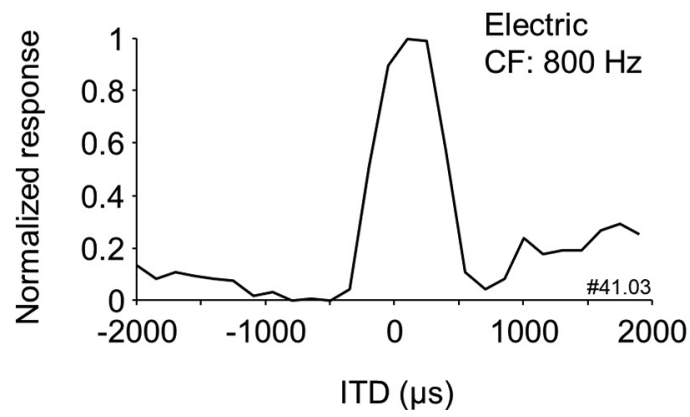

B

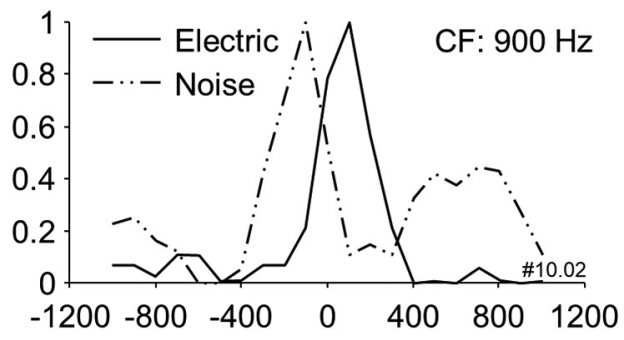

\section{Acoustic}
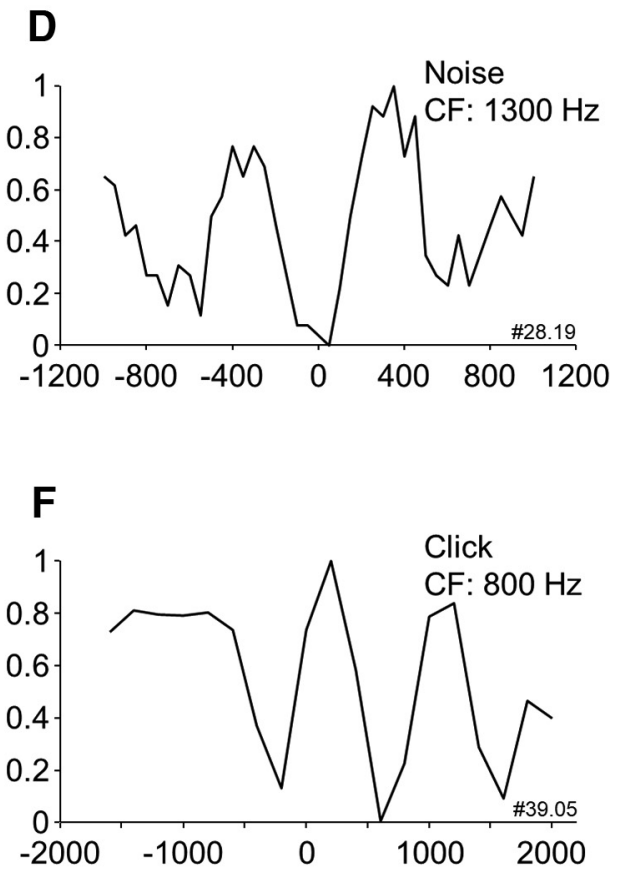

H

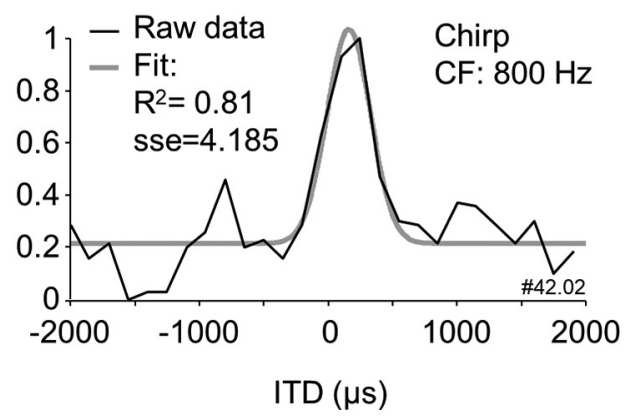

Figure 3. Differences in ITD functions for electric versus acoustic broadband stimuli are consistent with electroneural activation by electric pulses. $\boldsymbol{A}$, $\boldsymbol{B}$, ITD functions of two single neurons for electric pulses and acoustic noise each. ITD functions for electric pulses show only a single peak, whereas those for acoustic noise can have multiple peaks. $\boldsymbol{C}-\boldsymbol{H}$, ITD functions for three pairs of neurons with similar $(F s(C / D, E / F, G / H)$ for electric and broadband acoustic stimuli. ITD functions for electric pulses display a single peak or trough $(\boldsymbol{C}, \boldsymbol{E}, \boldsymbol{G})$, consistent with electroneural but not with electrophonic activation. In contrast, ITD functions for noise $(\boldsymbol{D})$, clicks $(\boldsymbol{F})$, and chirps $(\boldsymbol{H})$ can have multiple peaks. Stimulus type and $(F$ are listed for each neuron. Gray function in $\boldsymbol{H}$ displays $\mathbf{G a u s s i a n}$ fit to the central peak of the ITD function. 
A

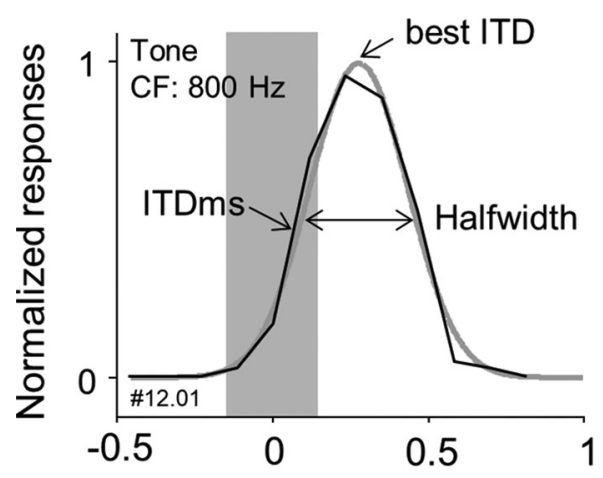

C

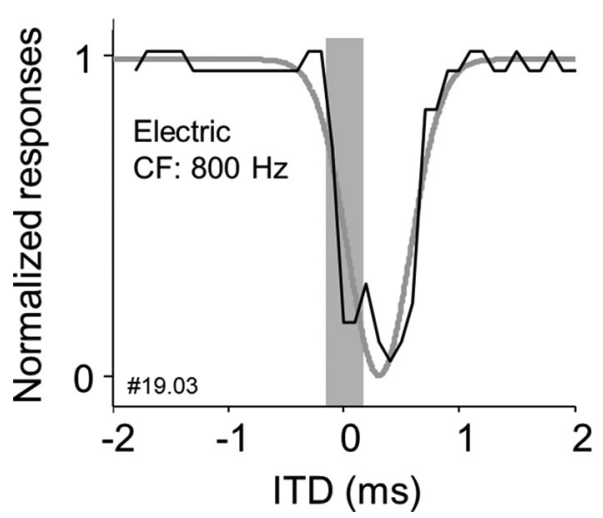

B

Biphasic

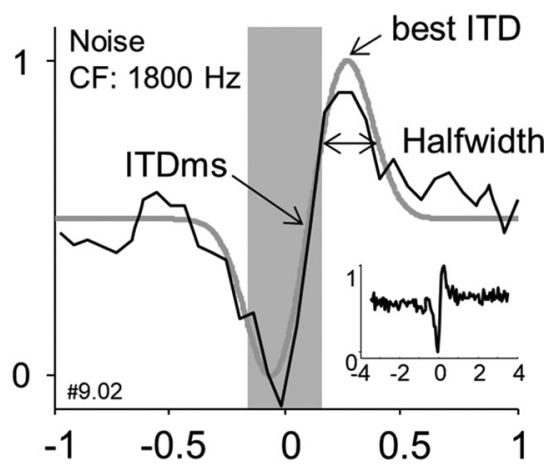

D Sigmoid

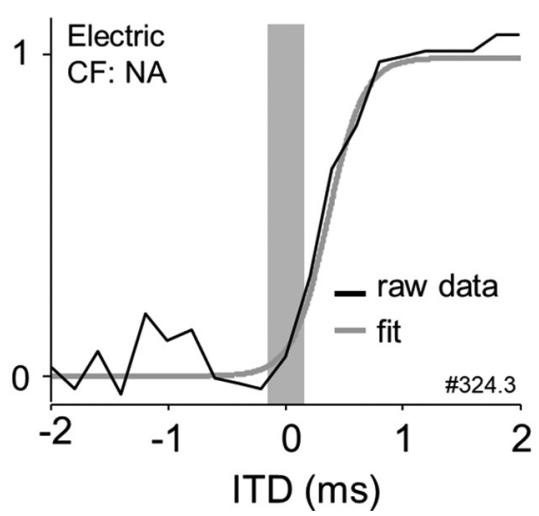

Figure 4. Shapes of ITD functions vary across neurons. Shape categorization of raw data (black functions) was based on four fitting functions (gray) as follows. A, Peak shape (positive Gaussian function; data are identical to the central peak at $\mathrm{BF}_{\mathrm{ITD}}, 900 \mathrm{~Hz}$, in Fig. 1A). $\boldsymbol{B}$, Biphasic shape (difference of two Gaussians). Inset shows the ITD function over an extended ITD range. $\boldsymbol{C}$, Trough shape (negative Gaussian). $\boldsymbol{D}$, Sigmoid shape for monotonic ITD functions. The fits for peak shape $(\boldsymbol{A})$ and biphasic ITD functions $(\boldsymbol{B})$ were used to determine the parameters of interest: best ITD (ITD at maximum response); half-width (ITD range at half-height); ITDms (ITD at maximum slope) (for details, see "ITD tuning" section in Materials and Methods). Gray area indicates the physiological ITD range in the gerbil ( $\pm 135 \mu \mathrm{s}$ ). Each fit was normalized to its minimum and maximum. The stimuli used to measure ITD tuning and the neuron's $C F$ are noted in each panel. CF was not available for the neuron in $\boldsymbol{D}$.

ies found trough-type neurons in the MSO (Day and Semple, 2011) and intermediate-type neurons in the MSO and LSO (Batra et al., 1997) and therefore call into question a simple association between response types and underlying mechanisms.

The distribution of ITD response types is shown in Figure $5 B$. Peak-type neurons were most common (56/126, 44\%), followed by intermediate-type neurons $(36 / 129,29 \%)$ and trough-type neurons $(34 / 126,27 \%)$. The dominance of peak-type neurons is consistent with previous studies of pure tone ITD processing in DNLL and IC (Kuwada et al., 2006; Siveke et al., 2006).

In Figure $5 C$, the shapes of ITD functions in response to the different broadband stimuli were related to the neurons' corresponding ITD response types to tones. Because the distributions of shape categories versus response types were similar for clicks and chirps, data for these two transient acoustic stimuli were pooled. For neurons with peak-shape ITD functions, the proportions of corresponding tone-evoked ITD response types were similar across the three groups of broadband stimuli ( $\chi^{2}$ test, $p>$ $0.05)$ : For all three broadband stimuli, $52-57 \%$ of neurons with peak-shape ITD functions were associated with peak-type neurons, $25-30 \%$ were associated with trough-type neurons, and $19-22 \%$ with intermediate-type neurons.

Even if data for peak-type and intermediate-type neurons were pooled and peak-shape functions were combined with bi-
Table 1. Statistical comparisons of CF, ITD tuning metrics, and NDTs for electric and acoustic stimulation between DNLL and IC

\begin{tabular}{|c|c|c|c|c|c|}
\hline Stimulus & DNLL & $N$ & IC & $N$ & $p$ \\
\hline \multicolumn{6}{|l|}{$\mathrm{CF}(\mathrm{Hz})$} \\
\hline Electric pulses & $900 \pm 341$ & 25 & $800 \pm 375$ & 33 & 0.21 \\
\hline Click/chirp & $800 \pm 413$ & 21 & $700 \pm 230$ & 37 & 0.43 \\
\hline Noise & $900 \pm 325$ & 43 & $900 \pm 300$ & 55 & 0.13 \\
\hline Tone (peak only) & $900 \pm 200$ & 70 & $900 \pm 255$ & 62 & 0.39 \\
\hline \multicolumn{6}{|l|}{ Best ITD ( $\mu s)$} \\
\hline Electric pulses & $129 \pm 111$ & 22 & $115 \pm 120$ & 25 & 0.68 \\
\hline Click/chirp & $166 \pm 71$ & 19 & $142 \pm 47$ & 28 & 0.63 \\
\hline Noise & $163 \pm 74$ & 35 & $185 \pm 90$ & 40 & 0.9 \\
\hline Tone (peak only) & $185 \pm 156$ & 21 & $130 \pm 107$ & 28 & 0.38 \\
\hline \multicolumn{6}{|l|}{ Half-width $(\mu s)$} \\
\hline Electric pulses & $290 \pm 218$ & 22 & $470 \pm 173$ & 25 & 0.09 \\
\hline Click/chirp & $535 \pm 227$ & 19 & $468 \pm 159$ & 28 & 0.58 \\
\hline Noise & $375 \pm 130$ & 37 & $376 \pm 113$ & 40 & 0.82 \\
\hline Tone (peak only) & $493 \pm 218$ & 21 & $534 \pm 173$ & 28 & 0.66 \\
\hline \multicolumn{6}{|l|}{ ITDms ( $\mu s)$} \\
\hline Electric pulses & $-37 \pm 34$ & 22 & $-1 \pm 78$ & 25 & 0.32 \\
\hline Click/chirp & $-157 \pm 145$ & 19 & $-50 \pm 96$ & 28 & 0.35 \\
\hline Noise & $-30 \pm 104$ & 37 & $-2 \pm 54$ & 42 & 0.88 \\
\hline Tone (peak only) & $-52 \pm 160$ & 21 & $-46 \pm 95$ & 28 & 0.68 \\
\hline \multicolumn{6}{|l|}{$\mathrm{NDT}(\mu \mathrm{s})$} \\
\hline Electric pulses & $73 \pm 77$ & 21 & $129 \pm 63$ & 23 & 0.24 \\
\hline Click/chirp & $129 \pm 62$ & 18 & $111 \pm 42$ & 28 & 0.94 \\
\hline Noise & $93 \pm 65$ & 32 & $114 \pm 42$ & 37 & 0.3 \\
\hline Tone (peak only) & $117 \pm 70$ & 19 & $117 \pm 70$ & 25 & 0.92 \\
\hline
\end{tabular}

Data were determined from peak-shape and biphasic ITD functions for electric pulses, acoustic clicks/chirps, and noise and from peak-type neurons in response to tones. Values are given as median $\pm Q$. Statistical comparisons were performed using the Mann-Whitney rank-sum test. $N$, Number of neurons. 
A

Shape category

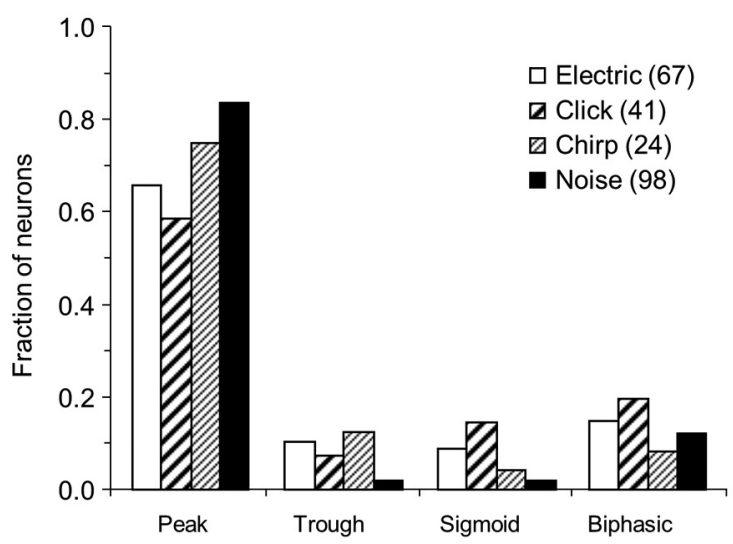

C Shape category vs. Response type
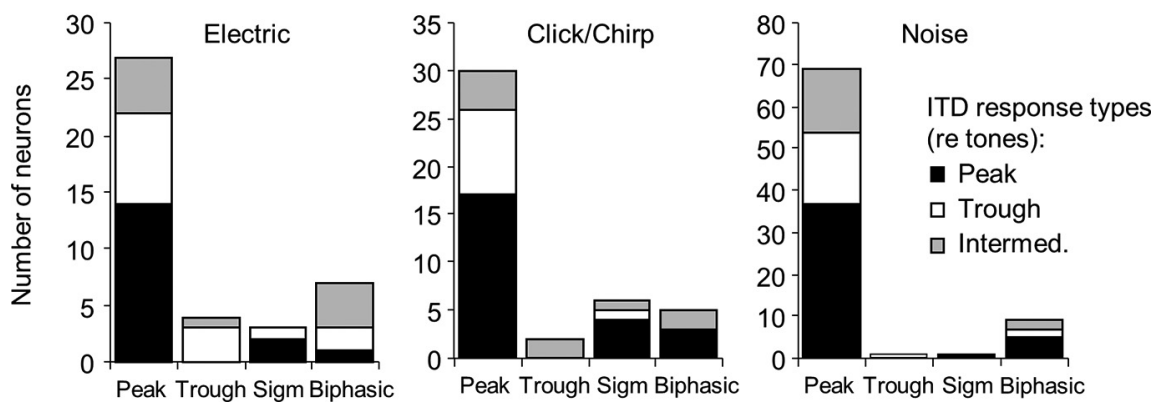

Figure 5. Distributions of ITD shape categories and response types. A, Fractions of the four different ITD tuning shapes for broadband stimuli of either mode (electric, acoustic). Numbers in brackets indicate the number of neurons. $\boldsymbol{B}$, Fractions of ITD response types for pure tone stimulation. C, Distributions of shape categories of single neurons in response to electric pulses (left), clicks/chirps (middle), and noise (right) are separately displayed for corresponding ITD response-type categories (peak, trough, and intermediate) of the same neurons in response to tone stimulation.

phasic functions (data not shown), the results indicate that, independent of the stimulus mode or the temporal properties of the acoustic stimuli, the shapes of only $\sim 2 / 3(67 \%)$ of the ITD functions for broadband stimuli predict the corresponding ITD response type for tonal stimulation.

\section{ITD tuning metrics}

To characterize and compare neuronal ITD tuning across the different stimulus types quantitatively, three tuning metrics were derived from the fits of peak shape and biphasic ITD functions: best ITD, half-width of ITD tuning, and ITDms (Fig. 4A,B). For tones, only tuning metrics from peak-type neurons were included for comparison (see Materials and Methods). The characterization of ITD tuning metrics for tonal stimulation was based on the peak fit to the most central peak of the function at $\mathrm{BF}_{\text {ITD }}$ (Figs. 1A, 4A).

\section{DNLL versus IC}

The proportion of ITD-sensitive neurons for any given stimulus did not differ between DNLL and IC (all $>73 \%$; $\chi^{2}$ test, all $p>$ 0.09 ). Across all stimulus types, there were no differences in ITD tuning between neurons in the DNLL and the IC for any of the metrics tested (see Table 1; Mann-Whitney rank-sum test, all $p>$ 0.09). The results for best ITD, half-width, and ITDms were therefore pooled across the two nuclei for further analyses.
Best ITD

Figure $6 A$ shows the distributions and cumulative probabilities of best ITDs for the five stimulus types (electric pulses, acoustic clicks, chirps, noise, and tones). The median best ITDs are marked by the intersections of the cumulative probabilities with the horizontal lines. Across all stimulus types, best ITDs were biased toward positive values (contralateral leading). Most ITD functions for electric pulses (85\%), acoustic clicks (88\%), chirps (90\%), noise (90\%), and tones (70\%) peaked at positive ITDs. For all stimulus types, the peaks of the distributions fell between ITDs of +100 and $+200 \mu \mathrm{s}$. Moreover, most best ITDs for electric pulses (55\%), acoustic clicks (56\%), chirps (70\%), noise (60\%), and tones (57\%) lay outside of the physiologically relevant range of ITDs in gerbils ( $\pm 135 \mu \mathrm{m}$, gray area; Maki et al., 2003).

Box plots in Figure $6 B$ show the statistical comparisons of best ITDs for the same neurons presented in Figure $6 A$. For all five stimulus types, median best ITDs were outside the physiological range (gray area) and did not differ between stimulus types $(\mathrm{K}-\mathrm{W}, p=0.521)$. Also, the ANOVA accounting for random (neuronal) effects did not reveal significant differences in best ITDs across groups ( $p=$ 0.068).

Figure $6 C$ plots best ITDs for electric pulses versus transient acoustic broadband stimuli (pooled click/chirp responses) for a sample of 38 neurons. The data points scattered broadly around the diagonal, and best ITDs for the two stimulus modes were not significantly correlated (linear regression, $\left.R^{2}=0.007, p=0.274\right)$. In contrast, best ITD for tones and noise $(p<0.001, n=79)$ and best ITDs for clicks and noise $(p=0.008$, $n=22$ ) were significantly correlated (data not shown), confirming previous findings (Yin et al., 1986; Carney and Yin, 1989; Fitzpatrick et al., 2000).

Figure $6 D$ shows the corresponding matched-pairs comparison of best ITDs for electric pulses and acoustic clicks/chirps. Similar to the population analysis in Figure $6 B$, the withinneuron comparison also demonstrated a clear bias toward contralateral leading ITDs for the two modes of stimulation with the median best ITDs outside of the physiological range. Pairwise comparisons of electric and acoustic best ITDs determined in the same cells showed no significant difference in median best ITDs for the two stimulus modes (Wilcoxon signed-rank test, $p=$ 0.212; Fig. 6D).

\section{Best ITD versus $C F$}

In normal-hearing cats, gerbils, and guinea pigs, the best ITDs of low-frequency IC and MSO neurons to acoustic narrowband and broadband stimuli tend to be inversely correlated with their CFs (McAlpine et al., 2001; Hancock and Delgutte, 2004; Joris et al., 2006; Siveke et al., 2006; Plauška et al., 2017). Figure 7A-D, shows the relationship between the best ITDs and CFs of neurons that 

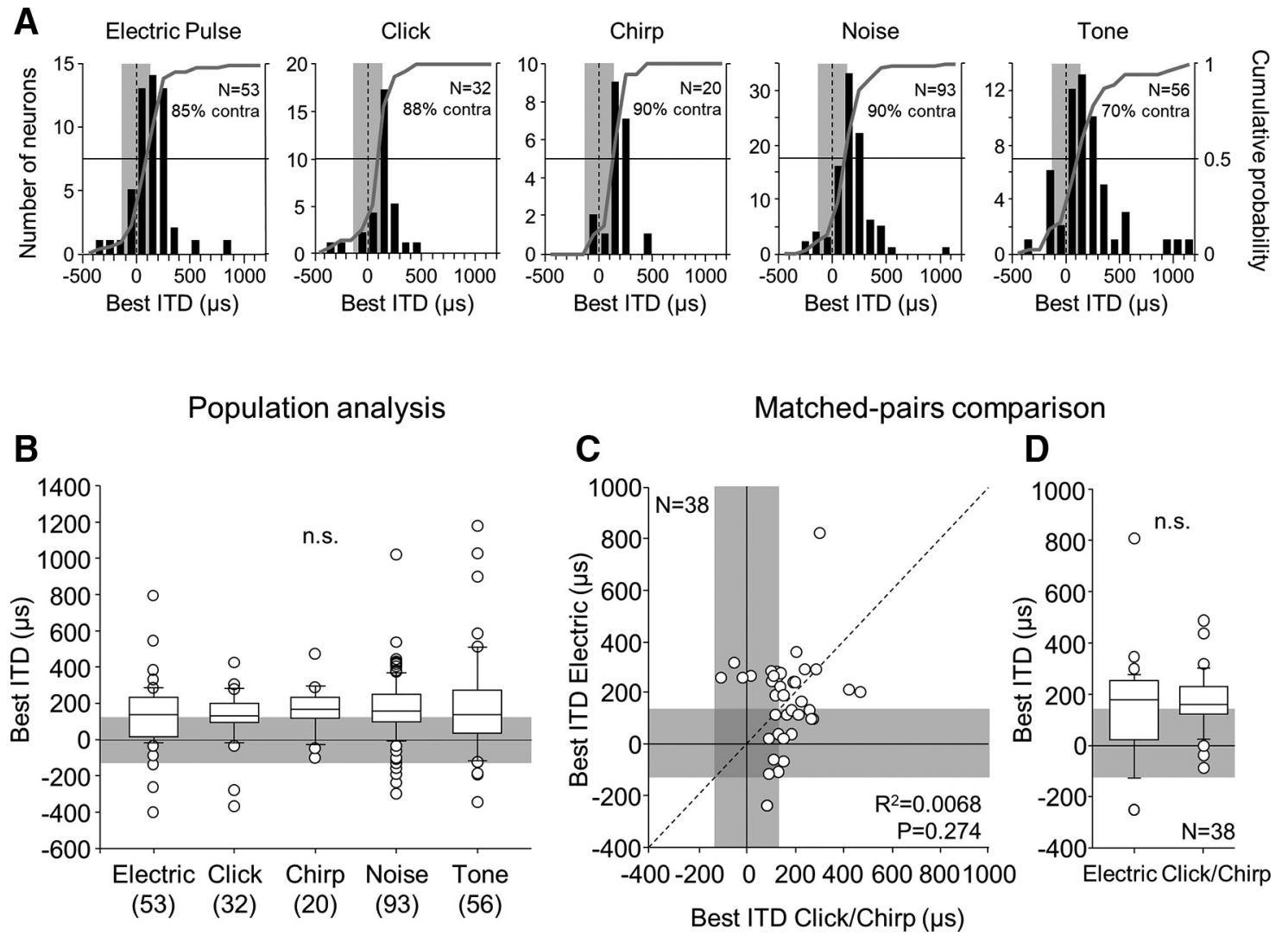

Figure 6. Populations of neurons have similar best ITDs for electric and acoustic stimulation. $A$, Distributions (black bars) and cumulative probabilities (gray functions) of best ITDs for electric pulses and acoustic clicks, chirps, noise, and pure tones. Dashed vertical line indicates zero ITD; horizontal line, cumulative probability of $0.5 ; \mathrm{N}$, number of neurons; contra, contralateral leading best ITDs. $\boldsymbol{B}$, Population statistics of best ITD for electric and acoustic stimuli for the same neurons as in $A$. n.s., Not significant (K-W, ANOVA accounting for random effects, both $p>0.05$ ). Numbers in parentheses are the number of ITD-sensitive neurons. C, Plot of best ITDs for electric pulses versus best ITDs for acoustic clicks/chirps of the same neurons. Dashed line indicates equal values. $\boldsymbol{D}$, Matched-pairs comparison between best ITDs for electric pulses and acoustic clicks/chirps for the same neurons as in C. n.s., Not significant (Wilcoxon signed-rank test, $p=0.212$ ). Gray bars in $\boldsymbol{A}-\boldsymbol{D}$ indicate the physiological ITD range in the gerbil.

were ITD sensitive to the different stimulus types. As in Figure 6, best ITDs for electric pulses (Fig. 7A), acoustic clicks/chirps (Fig. $7 B$ ), and noise stimuli (Fig. 7C) were derived from peak-shape and biphasic ITD functions. For tones, the sample includes peaktype and intermediate-type neurons (Fig. 7D). The dependence of best ITD on CF was quantified by dividing the neurons into quintiles based on CF and computing the mean absolute best ITD for each quintile (red circles). For all stimulus types, the majority of neurons had best ITDs within half a cycle of their CFs (dashed lines). Only for tone stimulation, a weak trend of increasing best ITD with decreasing CF was observed (Fig. $7 D$ ). However, a larger sample of low-frequency neurons $(\mathrm{CF}<400 \mathrm{~Hz})$ is needed to thoroughly assess the possibility of a CF dependence of best ITDs for broadband stimuli of either mode.

\section{Half-width}

Figure $8 \mathrm{~A}$ shows the distributions and cumulative probabilities of half-widths for the five stimulus types. More than $50 \%$ of the neurons had half-widths $<600 \mu$ s for clicks, chirps, and tones, whereas $>50 \%$ had half-widths $<450 \mu$ s for electric pulses and acoustic noise. Box plots in Figure $8 B$ show the corresponding statistical comparisons of half-widths. Both the K-W and the ANOVA accounting for random effect (after log transformation of the data) indicated significant differences in half-widths among the different groups (both $p<0.001$ ). Subsequent Bonferroniadjusted multiple $t$ tests with random effects showed that, independent of the stimulus mode, there were no significant differences in median half-widths between any of the broadband stimuli (all $p>$ $0.06)$. However, half-widths for electric pulses and noise were significantly narrower than for tones ( $p=0.008$ and $p=0.026$, respectively). This result is difficult to interpret because half-widths for tones are inversely correlated with tone frequency.

When recorded from the same neurons, half-widths of ITD functions for electric pulses and acoustic clicks/chirps scattered broadly around the diagonal and were not significantly correlated $\left(R^{2}=0.02, p=0.349 ;\right.$ Fig. $\left.8 C\right)$. In accordance with the population results in Figure $8 B$, the matched-pairs comparison showed no significant difference in median half-width for electric pulses and acoustic clicks/chirps (Wilcoxon signed-rank test, $p=0.392$; Fig. $8 D)$.

\section{ITDms}

Best ITD and half-width determine the ITDms, where changes in firing rate with changes in ITD are most pronounced. Consistent with the contralateral bias of best ITDs and the relatively narrow ITD tuning in normal hearing animals (Figs. 6, 8, respectively), distributions of ITDms for all stimulus types were centered around zero ITD, with the peaks of the distribution lying within the physiological ITD range in gerbil (Fig. 9A). Statistical comparisons in Figure $9 B$ demonstrated no differences in the median ITDms across stimulus types (K-W, $p=0.339$; ANOVA accounting for random effects, $p=0.611$ ). Median ITDms for all stimulus types lay within the physiological range and occurred at negative ITDs (ipsilateral leading). 

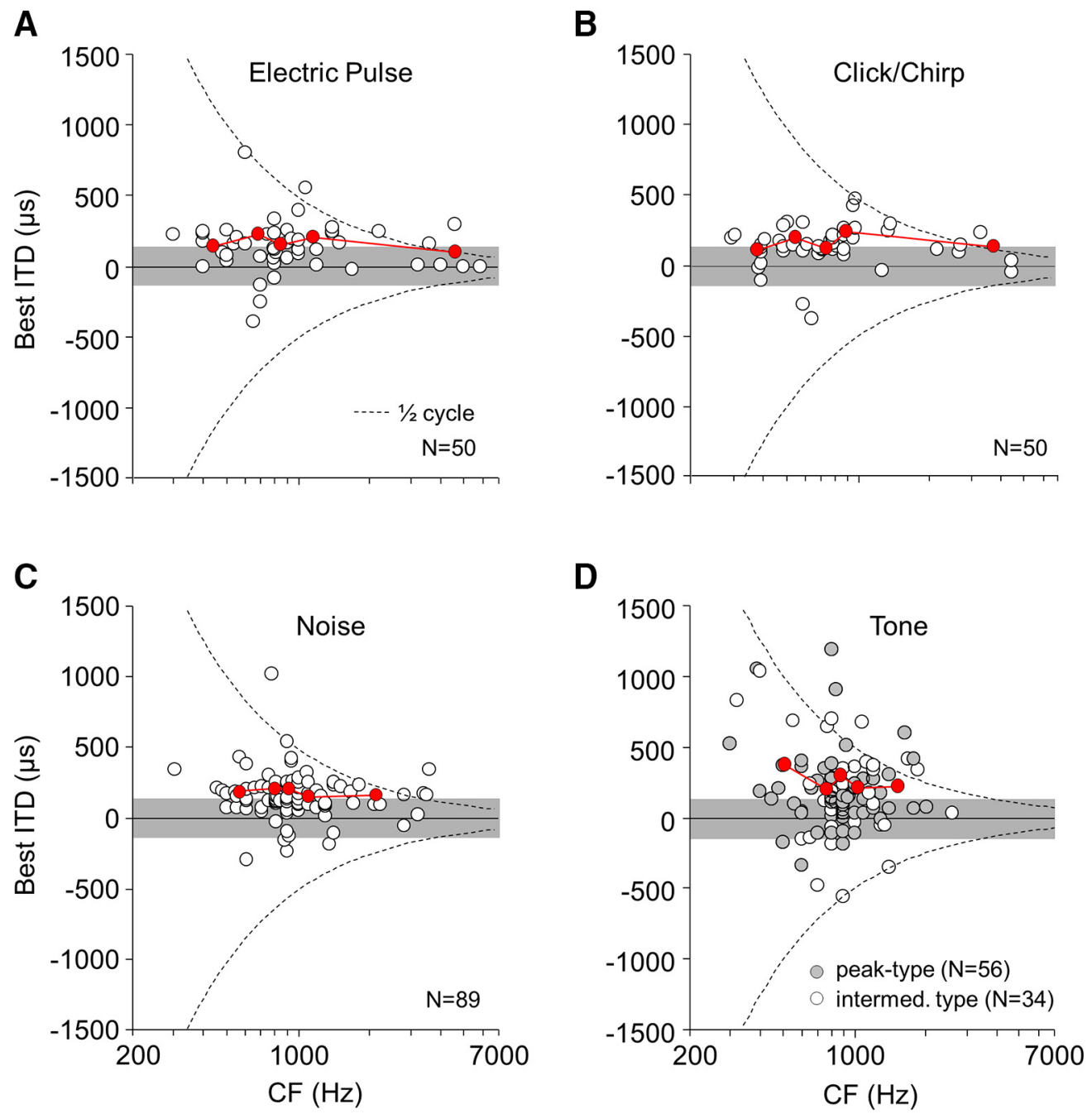

Figure 7. Best ITDs as a function of CF for neurons that were sensitive to ITDs of electric pulses $(\boldsymbol{A})$, acoustic clicks/chirps $(\boldsymbol{B})$, noise $(\boldsymbol{C})$, and pure tones $(\boldsymbol{D})$. For tones $(\boldsymbol{D})$, values are shown separately for peak-type (gray circle) and intermediate-type neurons (open circle). Red circles in $\boldsymbol{A}-\boldsymbol{D}$ show mean values of absolute best ITD values for each quintile of CFs. Dashed lines indicate half cycles of CF. Gray bars in $\boldsymbol{A}-\boldsymbol{D}$ indicate physiological ITD range in the gerbil. $N$, Number of single neurons.

Figure 9C directly compares ITDms for electric pulses and acoustic clicks/chirps for a sample of 40 neurons. Consistent with the findings for best ITD and ITD half-width (Figs. 6C, 8C), ITDms values for acoustic clicks/chirps and electric pulses scattered broadly around the diagonal and were not significantly correlated $\left(R^{2}=0.001, p=0.855\right)$. The matched-pairs comparison of ITDms values for electric pulses and acoustic clicks/chirps in this sample of neurons showed no significant difference between the two stimulus types (Wilcoxon signed-rank test, $p=0.386$; Fig. 9D).

\section{Population coding}

To qualitatively assess the relative effects of stimulus mode and spectrotemporal stimulus properties on ITD processing, we tested for differences in population coding across the different stimulus types. Normalized Gaussian best fits for individual ITD functions were averaged to obtain population ITD functions for each of the four stimulus types (Fig. 10). Higher peaks reflect a higher proportion of ITD functions with similar ITDs. Here, the ITD function for clicks/chirps demonstrates the highest peak due to the narrow distribution of best ITDs for these stimuli (Fig. $6 A, B)$. Overall, population ITD tuning was similar across all four stimulus types: best ITD had a pronounced contralateral bias and
ITD half-width was relatively narrow. As a result, all ITD functions had similarly steep slopes. Neural population coding is an essential prerequisite for the concept of a "two-channel" model of directional hearing. According to this model, directional hearing in the horizontal plane is based on a population code in which the relative activation of two populations of broadly tuned neurons is compared between the two brain hemispheres (McAlpine et al., 2001; McAlpine and Grothe, 2003; Stecker et al., 2005; Devore et al., 2009; Hancock et al., 2013; Stange et al., 2013) rather than on ITD tuning characteristics of (sharply tuned) individual neurons. Assuming that ITD discrimination in a two-channel model is proportional to the slope of the average ITD function, the observed similarity in population tuning suggests high acuity in ITD perception in normal-hearing animals that is independent of the stimulus mode or the spectrotemporal properties of the acoustic stimuli.

\section{Neural ITD NDTs}

To compare neural ITD acuity across the different stimulus types, NDTs were estimated for each cell and stimulus type by computing a modified version of the standard separation (Sakitt, 1973; Smith and Delgutte, 2007; see Materials and Methods) between each ITD step and a reference ITD. The NDT is defined as the ITD 
A

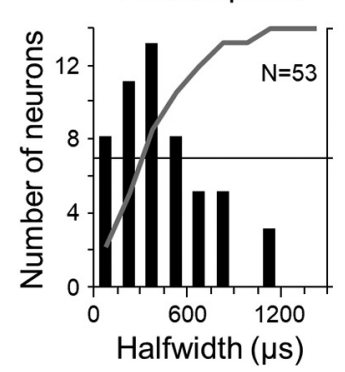

Click

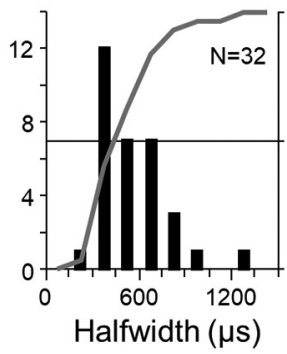

Chirp

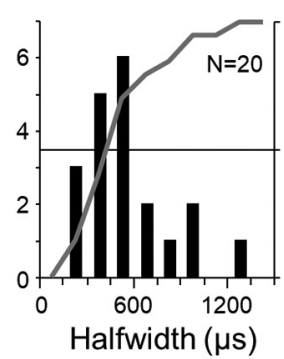

Noise

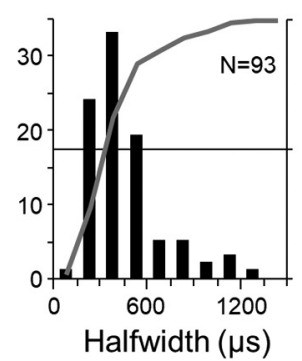

Tone

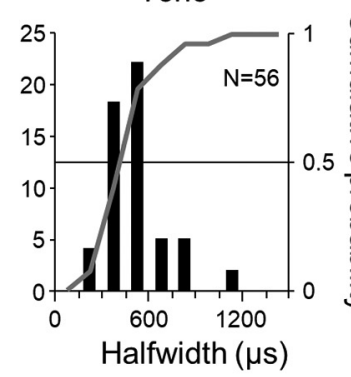

\section{Population analysis}

\section{B}

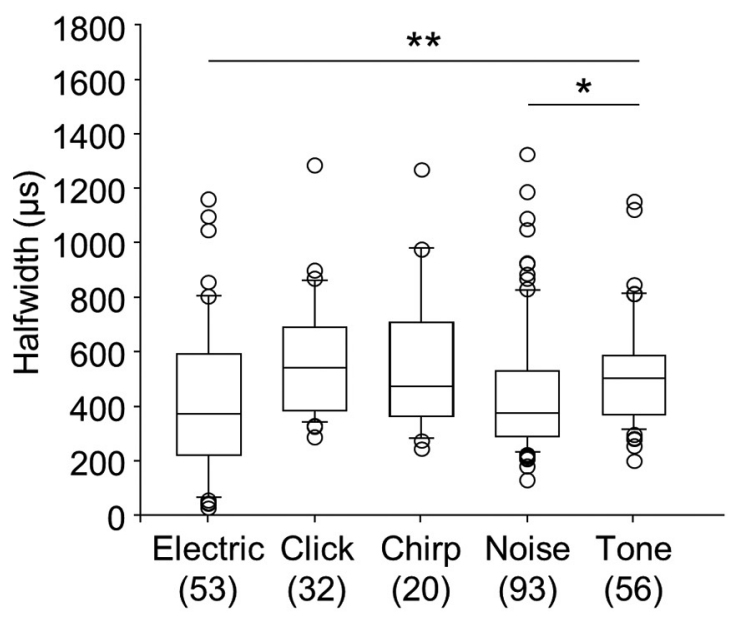

\section{C}

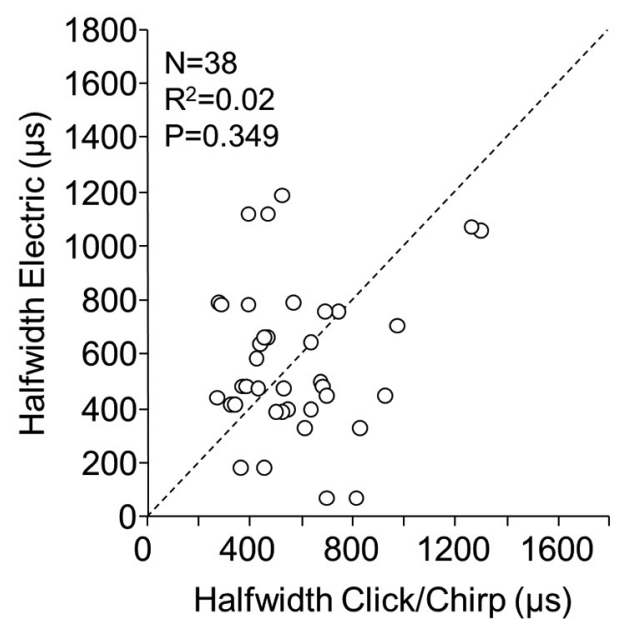

Matched-pairs comparison

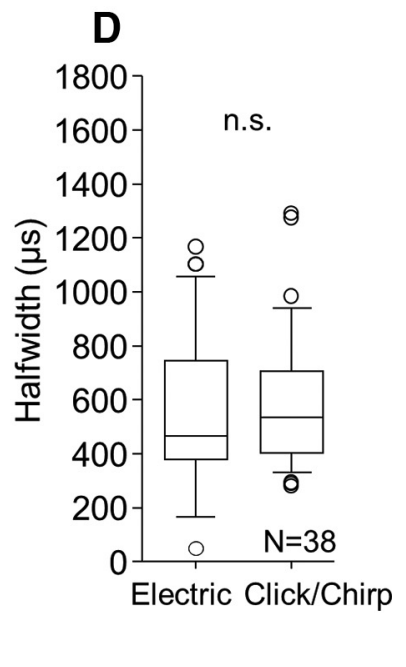

Figure 8. Population analysis shows similar half-widths for electric and acoustic broadband stimuli. $\boldsymbol{A}$, Distributions (black bars) and cumulative probabilities (gray functions) of half-widths for the five stimulus types. Horizontal line indicates the cumulative probability of $0.5 ; N$, number of neurons. $\boldsymbol{B}$, Population analysis of half-width for electric and acoustic stimuli for the same neurons as in $\boldsymbol{A}$ [K-W, ANOVA accounting for random effects (log data), both $p<0.001$; Bonferroni-adjusted multiple $t$ tests with random effects, $\left.{ }^{*} p=0.026,{ }^{* *} p=0.008\right]$. Numbers in parentheses are the number of neurons. $\boldsymbol{C}$, Correlation between neuronal half-widths for electric pulses and acoustic clicks/chirps. Dashed line indicates equal values. $\boldsymbol{D}$, Matched-pairs comparison between half-widths for electric pulses and acoustic clicks/chirps for the same neurons as in C. n.S., Not significant (Wilcoxon signed-rank test, $p=0.392$ ).

closest to the reference ITD for which the standard separation reaches a value of one.

In human studies, ITD discrimination is best for reference sounds in the front. Therefore, NDTs were calculated for a fixed reference of zero ITD (Fig. 11). There were no differences in NDTs between neurons in the DNLL and the IC for any of the stimulus types tested (Table 1; Mann-Whitney rank-sum test all $p>0.24)$. NDTs were therefore pooled across the two nuclei.

Median NDTs did not differ significantly across stimulus types (electric pulses: $98 \pm 67 \mathrm{Q} \mu \mathrm{s}$, click: $127 \pm 45 \mu \mathrm{s}$, chirp: $111 \pm 43 \mu \mathrm{s}$, noise: $112 \pm 52 \mu \mathrm{s}$, tone: $92 \pm 63 \mu \mathrm{s}$; K-W, $p=$ 0.614; Fig. 11A). After log transformation of the data, the ANOVA accounting for random effects also did not reveal significant differences between NDTs across stimulus types $(p=0.560)$.

Figure $11 B$ directly compares NDTs for electric pulses versus acoustic clicks/chirps for a sample of 35 neurons. Most values scattered broadly around the diagonal, and there was no significant correlation between NDTs for the two stimulus modes $\left(R^{2}=0.01, p=0.537\right)$. However, in agreement with the population results in Figure $11 A$, the matched-pairs comparison in the same sample of neurons revealed no differences in median NDTs between electric pulses and acoustic clicks/chirps (129 $\pm 72 \mathrm{Q} \mu \mathrm{s}$ and $112 \pm 60 \mathrm{Q} \mu \mathrm{s}$, respectively; Wilcoxon signed-rank test, $p=$ 0.199; Fig. 11C]. Therefore, on a population level, NDTs were similar across all stimulus types independent of the stimulus mode or the spectrotemporal characteristics of the acoustic stimuli. This similarity in ITD discrimination acuity across the different stimulus types is consistent with the similarity in population ITD tuning described in Figure 10.

\section{CF distribution}

To exclude the possibility that potential differences in ITD tuning and ITD discrimination thresholds have been obscured by differences in the distributions of CFs for the five stimulus types, the CFs to diotic stimulation with pure tones were determined for the majority of ITD-sensitive neurons (154/183, 84\%). The CFs ranged from 0.3 to $14 \mathrm{kHz}$ with a peak between 0.75 and $1 \mathrm{kHz}$ (Fig. 12C, inset). Because there were no differences in the CFs of DNLL and IC neurons for any of the four stimulus types tested (electric pulse, click/chirp, noise, tone; Mann-Whitney ranksum test, all $p>0.13$; Table 1 ), CFs were pooled across all neurons for further analysis.

Figure $12 \mathrm{~A}$ shows the distributions and cumulative probabilities of CFs for the five stimulus types. Data were restricted to those neurons that fulfilled the inclusion criteria for further analyses (peak shape and biphasic ITD functions for electric pulses, clicks, chirps, and noise; peak-type neurons for tones; see Materials and Methods). Neurons that were ITD sensitive to tones had maximum CFs of $\sim 2 \mathrm{kHz}$, whereas neurons with ITD sensitivity 
A

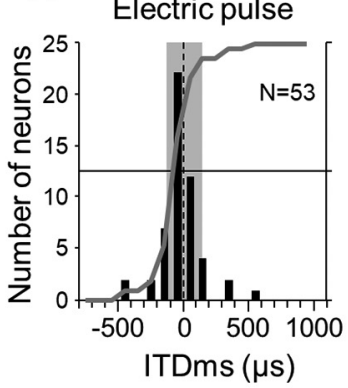

Click

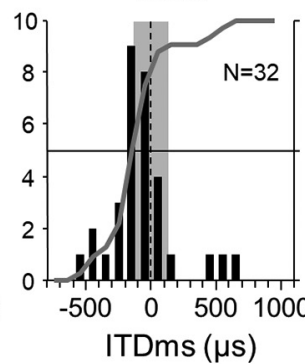

Chirp

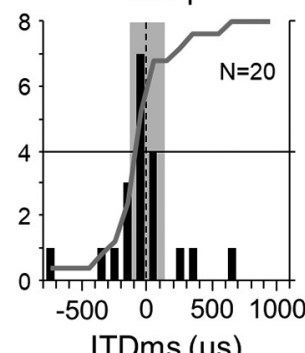

Noise

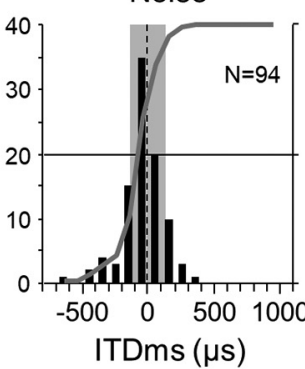

Tone

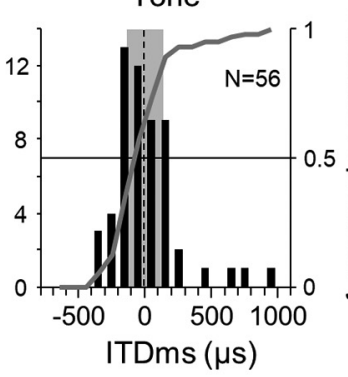

Matched-pairs comparison

B

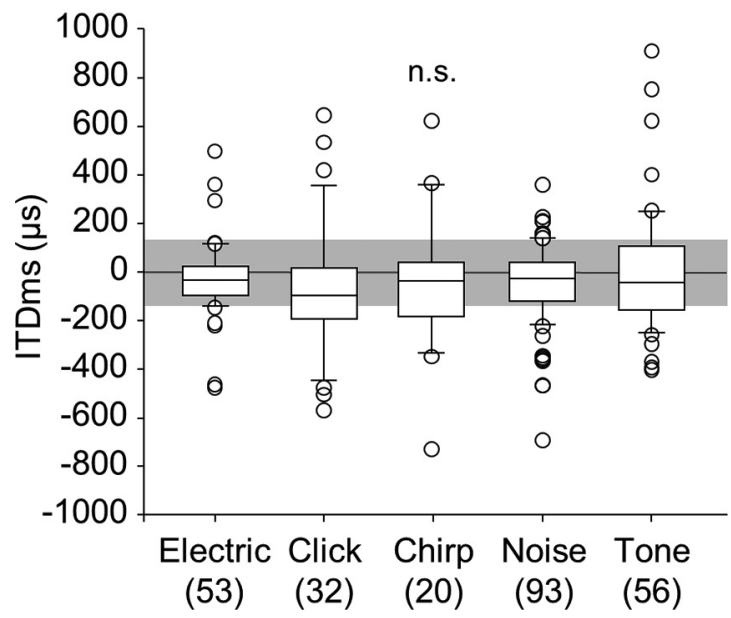

C

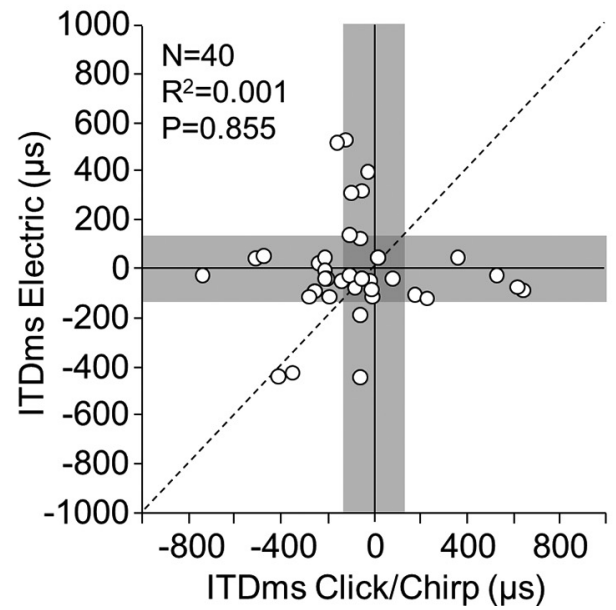

D

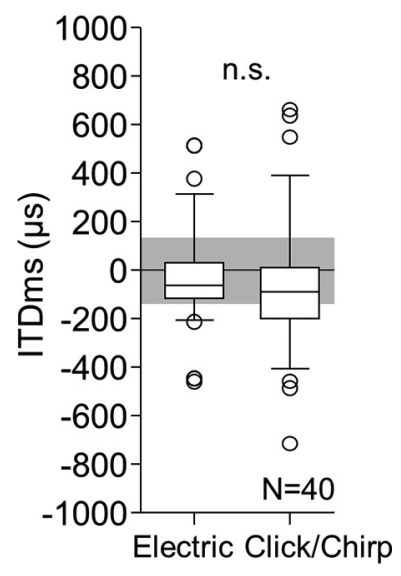

Figure 9. Population analysis shows similar ITDms for electric and acoustic stimuli. $\boldsymbol{A}$, Distributions (black bars) and cumulative probabilities ( $g$ ray functions) of ITDms values for the five stimulus types. Horizontal line marks the cumulative probability of 0.5 ; dashed vertical line, 0 ITD. $N$, Number of neurons. $\boldsymbol{B}$, Population statistics of ITDms across stimulus types for the same neurons as in $\boldsymbol{A}$. n.S., Not significant [K-W, $p=0.339$; ANOVA accounting for random effects, $p=0.611]$. Numbers in parentheses are the number of neurons. $C$, Comparisons of ITDms for electric pulses versus acoustic clicks/chirps. D, Matched-pairs comparison between ITDms values for electric pulses and acoustic clicks/chirps for the same neurons as in C. n.s., Not significant (Wilcoxon signed-rank test, $p=0.386)$. Gray bars in $\boldsymbol{A}-\boldsymbol{D}$ indicate the physiological ITD range in the gerbil.

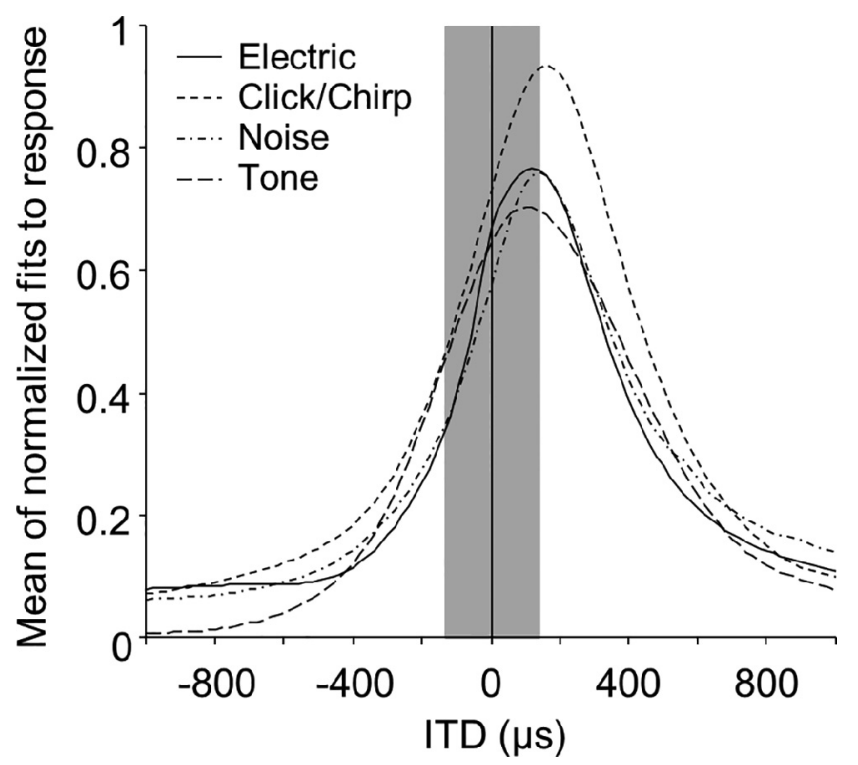

Figure 10. Population ITD coding of electric and acoustic stimuli is similar. Averaged ITD functions for the different stimulus types were computed from the normalized (minimum to maximum) Gaussian best fits. Gray bar indicates the physiological ITD range in the gerbil. to broadband stimuli had CFs extending to higher frequencies (maximum of $14 \mathrm{kHz}$ ). Independent of the stimulus mode or the spectrotemporal properties of the acoustic stimuli, $>2 / 3$ of all ITD sensitive neurons had CFs $<1 \mathrm{kHz}$, and the peak of all CF distributions was between $750 \mathrm{~Hz}$ and $1 \mathrm{kHz}$. Box plots in Figure $12 B$ show the corresponding statistical comparisons of CFs. There were no differences in median CFs across the different stimulus types $(\mathrm{K}-\mathrm{W}, p=0.116)$. Because some of the $\mathrm{CF}$ values for different stimulus types were derived from the same neurons, the samples were partially dependent. Therefore, differences in CFs were additionally tested for significance using an ANOVA that accounted for random (neuronal) effects after log transformation of the data (see Materials and Methods). Also, when accounting for random effects, the ANOVA did not demonstrate significant differences in the CFs across stimulus types ( $p=$ $0.359)$. These data show that potential differences in ITD tuning and ITD discrimination thresholds across stimulus modes and acoustic stimulus types were not obscured by differences in CFs.

In early experiments, neurons that were not ITD sensitive to tones were not always tested for ITD sensitivity to other stimuli (see Materials and Methods). This resulted in a sampling bias toward low CFs $(\leq 2 \mathrm{kHz})$. In addition, the medial-to-lateral orientation of the recording trajectory, which allowed recordings from both DNLL and IC in the same penetration, may 
Population analysis

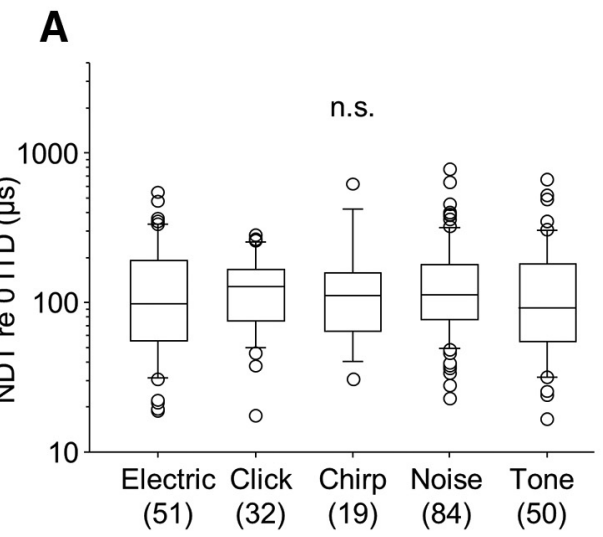

Matched-pairs comparison

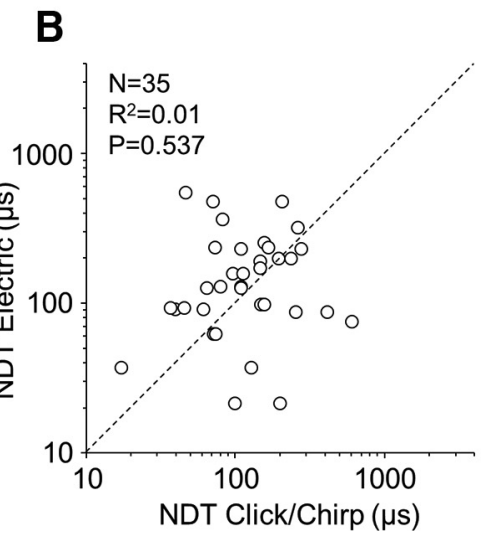

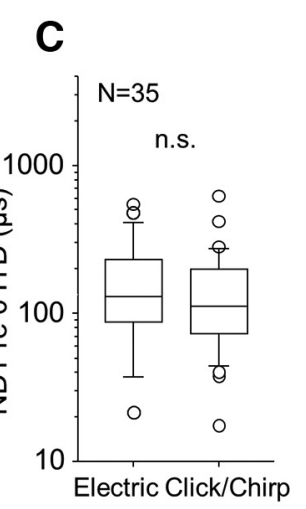

Figure 11. Neuronal populations have similar ITD discrimination thresholds (NDT, re zero ITD) for electric and acoustic stimulation. $\boldsymbol{A}$, Statistical comparisons of NDTs across the five stimulus types. n.s., Not significant [K-W, $p=0.614$; ANOVA accounting for random effects (log data), $p=0.560]$. Numbers in parentheses are the number of neurons. $\boldsymbol{B}$, NDTs for electric pulses and acoustic clicks/chirps were not significantly correlated (linear regression, $p=0.537$ ). $N$, Number of neurons. Dashed line indicates equal values. C, Matched-pairs comparison between NDTs for electric pulses and acoustic clicks/chirps for the same neurons as in B. n.S., Not significant (Wilcoxon signed-rank test, $p=0.199$ ). Gray bars in $\boldsymbol{A}-\boldsymbol{C}$ indicate the physiological ITD range in the gerbil.

have contributed to a low-CF bias (Hutson et al., 2018). A minority of neurons that were ITD sensitive to electric pulses (19\%), clicks/chirps (15\%), or noise (9\%) had high CFs $(>2$ $\mathrm{kHz}$ ). However, there were no significant differences in ITD tuning metrics and ITD discrimination thresholds between low- and high-CF neurons for any of the stimulus types tested (Mann-Whitney rank-sum test, $p>0.09$ for all comparisons), with the exception of half-widths for electric pulses (narrower for high-CF neurons, $p=0.032$ ) and for noise (broader for high-CF neurons, $p=0.024)$. Additional data recorded from high-CF neurons are required to more thoroughly evaluate the influence of CF on electric ITD processing.

The CFs of ITD-sensitive neurons were also compared with their $\mathrm{BF}_{\mathrm{ITD}}$ values, both obtained from responses to tonal stimulation (Fig. 12C). Results are shown separately for peak-type neurons (filled circles), intermediate-type and trough-type neurons (open circles), and for neurons that failed the criteria for response-type categorization (dashed circles; see "ITD tuning" section in Materials and Methods). For the majority of neurons, $\mathrm{BF}_{\mathrm{ITD}}$ values were lower than CFs. This difference was more pronounced for $\mathrm{CFs}>1 \mathrm{kHz}$. The difference in octaves between $\mathrm{CFs}$ and $\mathrm{BF}_{\mathrm{ITD}}$ values for CFs $>1 \mathrm{kHz}$ (median for all types $0.59 \pm$ 0.38 Q octaves) was significantly larger than that for CFs $\leq 1 \mathrm{kHz}$ $(0.15 \pm 0.18$ Q octaves; Wilcoxon signed-rank test, $p<0.001)$. These data are in accordance with previous reports showing that the majority of ITD-sensitive DNLL and IC neurons have $\mathrm{BF}_{\mathrm{ITD}}$ values $<1 \mathrm{kHz}$, and only few neurons had $\mathrm{BF}_{\mathrm{ITD}}$ values of up to 2 kHz (Fitzpatrick et al., 2000; Siveke et al., 2006).

\section{Discussion \\ Effects of spectrotemporal stimulus properties on acoustic ITD coding}

Acoustic stimuli with different spectrotemporal properties elicit different activation patterns across the population of ANFs that in turn may affect the convergence of inputs to higher-level neurons (Pfeiffer, 1966; Kiang et al., 1973; Kiang, 1975; Godfrey et al., 1975a,b; Bourk, 1976; Carney and Yin, 1988) and thus likely influence ITD processing of DNLL and IC neurons. When restricted to the central range of ITDs, the results of the present study revealed no differences in ITD tuning and ITD discrimination thresholds across acoustic stimuli with different spectrotemporal properties (Figs. $6 B, 9 B, 10,11 A$ ), with the exception of half-width (Fig. $8 B$ ). These findings are in agreement with prior studies demonstrating similarities in the sensitivities of lowfrequency MSO and IC neurons to ITDs of acoustic narrowband and broadband stimuli at moderate stimulus levels (Geisler et al., 1969; Yin et al., 1986; Caird and Klinke, 1987; Carney and Yin, 1989; Plauška et al., 2017).

\section{Population-based acoustic and electric ITD processing}

In animals with prior normal hearing experience, the stimulus mode (acoustic vs electric) had no effect on the overall nature of ITD processing (Figs. $6 B, 8 B, 9 B, 10,11 A$ ). On the population level, ITD tuning metrics and ITD discrimination thresholds of neurons with comparable CFs were largely similar for acoustic and electric stimulation. Independent of the stimulus mode, the majority of neurons displayed peak-shape ITD functions (Fig. $5 A, C)$ and responded maximally to ITDs around the contralateral edge of the physiological range. This bias toward contralaterally leading best ITDs (Fig. 6) is consistent with previous studies on ITD processing of acoustic narrowband and broadband stimuli in different auditory brainstem and midbrain nuclei in gerbils (Spitzer and Semple, 1995; Seidl and Grothe, 2005; Siveke et al., 2006; Pecka et al., 2008; Belliveau et al., 2014; Plauška et al., 2017). For all stimulus types tested, the majority of DNLL and IC neurons (55-70\%) had best ITDs outside of the physiologically relevant range (Figs. 6, 7). These results are in agreement with prior acoustic studies in gerbil MSO and DNLL (Brand et al., 2002; Seidl and Grothe, 2005). However, other studies reported either clearly higher fractions of best ITDs outside the physiological range ( $>80 \%$; Pecka et al., 2008) or, in contrast, found that the majority of best ITDs fell within the physiological range (Day and Semple, 2011; Plauška et al., 2017). The variability in these findings may reflect the susceptibility of best ITD to a variety of stimulus factors such as level, bandwidth, or tone frequency, or it may be due to small sample sizes, especially for MSO recordings. Nevertheless, independent of differences in stimulus mode or spectrotemporal stimulus properties, all of these reports underscore the robust contralateral bias of gerbil best ITDs that allows spike rate to unambiguously encode ITDs over a large proportion of the physiological range or over its entire range (Fig. 10). Together, the overall similarities in the processing of ITDs of electric pulses, acoustic tones, clicks, chirps, and noise by DNLL and IC neurons support the hy- 
A
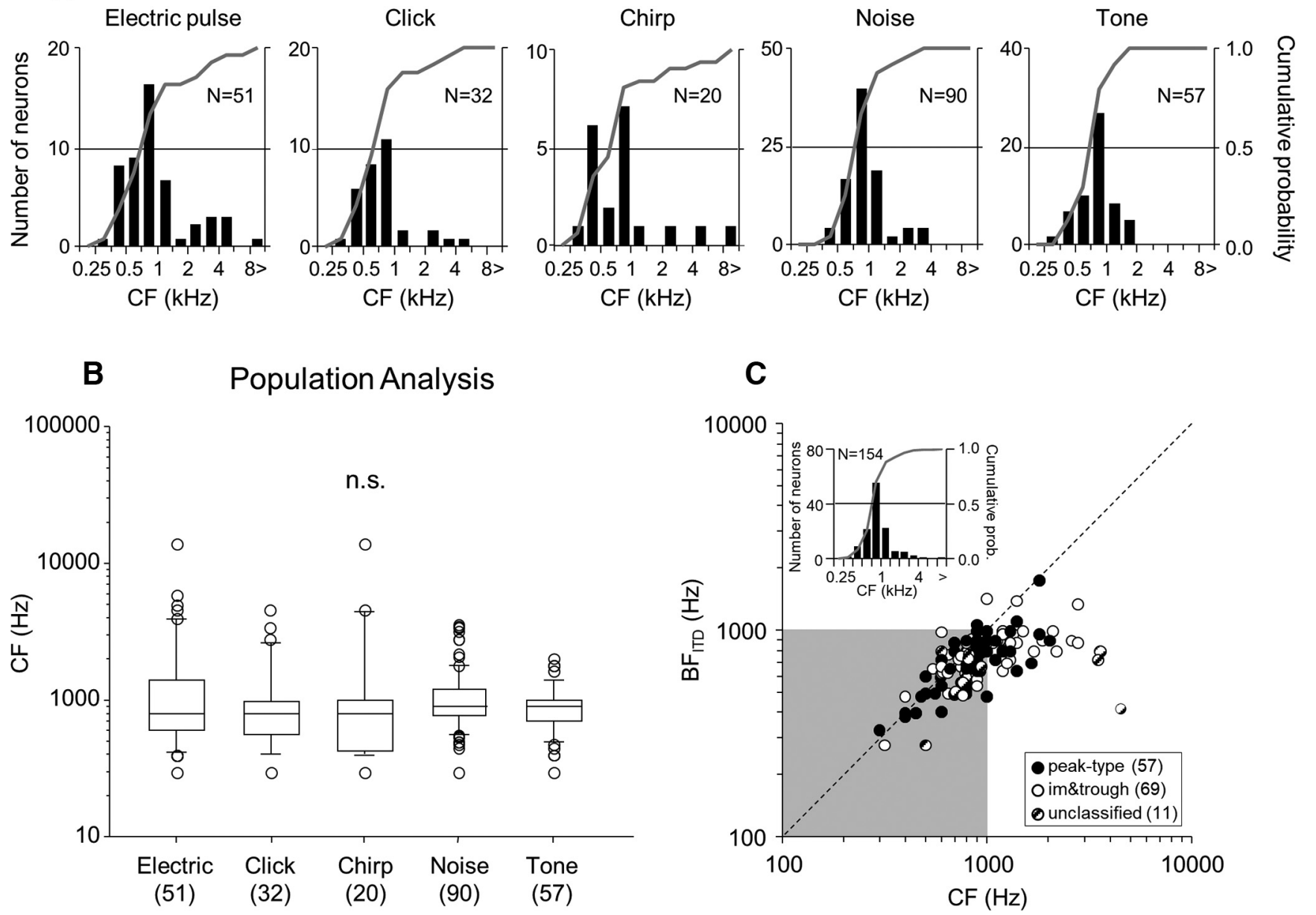

Figure 12. Distributions of CFs are similar for the different stimulus types. A, Distributions (black bars, left ordinate) and cumulative probabilities (gray functions, right ordinate) of CFs of neurons that were ITD sensitive to electric pulses and acoustic clicks, chirps, noise, and pure tones. Horizontal lines indicate a cumulative probability of 0.5. N, Number of neurons. $\boldsymbol{B}$, Population statistics of the (Fs of ITD-sensitive neurons in response to electric and acoustic stimuli (same neurons as in $A$ ). Graphs plot the median, 10th, 25th, 75th, and 90th percentiles of the (Fs as vertical boxes with error bars. A given neuron may appear in different groups if it was ITD sensitive to more than one stimulus. n.s., Not significant [K-W and ANOVA accounting for random neuronal effects (log data), both $p>0.05$ ]. C, Comparison between $\mathrm{BF}_{\text {ITD }}$ and CF for ITD-sensitive single neurons in response to tones. Filled circles indicate peak-type neurons; open circles, intermediate-type (i.m.) and trough-type (trough) neurons; dashed circles, neurons without response-type categorization (unclassified). Gray area indicates $\mathrm{BF}_{\text {ITD }}$ and $\mathrm{CF}$ values $\leq 1000 \mathrm{~Hz}$. Dashed line indicates equal values. Inset shows the distribution of CFs of the neurons tested with pure tones. Numbers in parentheses are the number of neurons.

pothesis that population ITD coding can be largely generalized across stimuli of different modes and spectrotemporal properties when measured in the central range of ITDs (Carney and Yin, 1989). The similarities in ITD coding further suggest that both acoustic and electric ITDs engage similar processing mechanisms and binaural brain circuits in the normal hearing auditory system.

In normal-hearing animals, ITD response types to tones are often thought to be associated with specific neural mechanisms underlying ITD coding. Specifically, peak-type neurons are associated with excitatory-excitatory coincidence mechanisms in the MSO and trough-type neurons with excitatory-inhibitory mechanisms, as in the LSO (for review, see Grothe and Pecka, 2014). This simple dichotomy between response types and underlying mechanisms has been questioned by studies that reported trough-type neurons in gerbil MSO (Day and Semple, 2011) and intermediate-type neurons in rabbit MSO and LSO (Batra et al., 1997). In the present study, the majority of ITD functions for broadband stimuli of either mode were peak-shaped (Fig. $5 A, C$ ). However, in at least a third of the cases, ITD response types to tones did not predict the shapes of ITD functions for broadband stimuli of either mode (Fig. 5C). These results suggest that, independent of the stimulus mode, there is no simple association between the shapes of ITD functions and the underlying binaural mechanism. Also, the observation that the shape of ITD functions can change with stimulus level (Benevento and Coleman, 1970; Carney and Yin, 1989; Hancock et al., 2013) argues against a simple relationship.

The mechanisms underlying the dominance of contralateralleading best ITDs are controversially discussed. The stereausis model (Shamma et al., 1989) proposes asymmetric delays caused by the traveling wave along the basilar membrane due to small between-ear mismatches in frequency tuning of individual MSO neurons. Direct electric stimulation of the auditory nerve bypasses the traveling wave and thus eliminates cochlear delays. However, the maintained contralateral bias in electric ITD tuning contradicts the proposal of cochlear delays as the main source of internal delays and supports other mechanisms, such as contralateral leading inhibition (Brand et al., 2002), asymmetric excitatory synaptic dynamics (Jercog et al., 2010), and axonal delays (Jeffress, 1948; Plauška et al., 2017). 


\section{Within-neuron comparisons of acoustic and electric ITD processing}

In agreement with prior findings (Yin et al., 1986; Carney and Yin, 1989; Fitzpatrick et al., 2000), within-neuron comparisons showed that best ITDs for tones and noise and for clicks and noise were significantly correlated. In contrast, ITD tuning and discrimination thresholds for acoustic clicks/chirps did not predict those for electric pulses. These results are consistent with recent recordings from MSO cells that showed differences in best ITD for acoustic broadband stimuli and electric pulses without displaying a preferred direction of mismatch (Plauška et al., 2017). Possible explanations for the observed nonsystematic discrepancies in ITD processing are differences in the temporal and spectral response patterns that are elicited across the population of ANFs by either stimulus mode. Electric stimulation bypasses cochlear filtering and traveling wave delays. Phase locking of ANFs to electric stimulation is much sharper than to acoustic stimulation (Dynes and Delgutte, 1992; Javel and Shepherd, 2000). Using biphasic electric pulses, the site of spike generation along the ANF (peripheral vs central axon) can affect spike timing (van den Honert and Stypulkowski, 1984; Miller et al., 1999; Shepherd and Javel, 1999; Joshi et al., 2017). Moreover, between-ear mismatches of electrode position, activation site, or relative stimulus level can lead to discharge patterns across the auditory nerves that differ in their spectral (CF) spread, firing rates, dispersion of latencies, and degree of synchronization between the two sides. These differences in peripheral spectrotemporal response patterns can affect the relative timing, strength, and balance of inhibitory and excitatory inputs converging on higher-level binaural neurons (Carney and Yin, 1989; He et al., 2012; Hancock et al., 2013; Hu and Dietz, 2015; Undurraga et al., 2016) and can thereby account for the observed within-neuron differences in ITD tuning and discrimination thresholds in response to the two modes of stimulation.

\section{Electric ITD coding in deafness}

In contrast to the present study, Smith and Delgutte (2007) reported significant differences in ITD tuning (best ITD and ITDms) for acoustic noise in normal-hearing cats and for electric pulses in acutely deafened adult cats with short deafness durations of 1-2 weeks. The CFs of the individual IC neurons were estimated based on the insertion depth of the recording electrode. The uncertainty of this method leaves the possibility that differences in the neurons' CFs contributed to the differences in ITD tuning between the two stimulus modes. In addition, the rapid changes in inhibitory synaptic strength that have been observed in the auditory brainstem and the IC after peripheral deafening (Takesian et al., 2009; Bender and Trussell, 2011) may underlie the altered shapes and tuning metrics of ITD functions for electric stimulation in animals with short deafness duration (Smith and Delgutte, 2007; Chung et al., 2016).

A number of recent studies in humans and animals suggest that temporal information is more accurately conveyed by electric stimulation of low-CF (apical) than high-CF (basal) regions of the cochlea and underscore the importance and effectiveness of low-CF electric activation (Krumbholz et al., 2000; Hamzavi and Arnoldner, 2006; Middlebrooks and Snyder, 2010; Macherey et al., 2011; Landsberger et al., 2014, 2016; Schatzer et al., 2014; Stahl et al., 2016). Additional data recorded from high-CF neurons are required to more thoroughly assess the influence of CF on neural ITD processing of transient electric and acoustic stimuli.

\section{ITD processing in DNLL versus IC}

The basic features of neural ITD processing (shape distributions of ITD functions, ITD tuning metrics, and neural ITD discrimination thresholds; Table 1) were similar in DNLL and IC independent of the stimulus mode or the spectrotemporal properties of the acoustic stimuli. These results are consistent with previous studies on acoustic ITD processing in unanesthetized rabbits except for a sharpening of ITD tuning in the IC observed by $\mathrm{Ku}-$ wada et al. (2006). The similarity in ITD processing between the two structures suggests that projections to DNLL and IC from primary ITD-processing structures (MSO and LSO) (BrunsoBechtold et al., 1981; Glendenning and Masterton, 1983) and projections from DNLL to IC are similarly engaged by the two stimulus modes.

\section{Conclusions}

Population-based ITD coding in the normal-hearing system was similar for electric and acoustic stimulation when measured in the central range of ITDs. The results suggest that differences in ITD discrimination between bilateral CI users and normalhearing listeners are primarily due to deafness-induced changes in neural ITD processing rather than to differences in the binaural brain circuits activated by electric versus acoustic ITDs. These findings support the idea that temporally correlated binaural hearing experience early in life and after short deafness durations is beneficial for ITD discrimination in binaural electric hearing.

\section{References}

Aronoff JM, Yoon YS, Freed DJ, Vermiglio AJ, Pal I, Soli SD (2010) The use of interaural time and level difference cues by bilateral cochlear implant users. J Acoust Soc Am 127:EL87-EL92. CrossRef Medline

Batra R, Kuwada S, Fitzpatrick DC (1997) Sensitivity to interaural temporal disparities of low- and high-frequency neurons in the superior olivary complex. I. Heterogeneity of responses. J Neurophysiol 78:1222-1236. CrossRef Medline

Belliveau LA, Lyamzin DR, Lesica NA (2014) The neural representation of interaural time differences in gerbils is transformed from midbrain to cortex. J Neurosci 34:16796-16808. CrossRef Medline

Bender KJ, Trussell LO (2011) Synaptic plasticity in inhibitory neurons of the auditory brainstem. Neuropharmacology 60:774-779. CrossRef Medline

Benevento LA, Coleman PD (1970) Responses of single cells in cat inferior colliculus to binaural click stimuli: combinations of intensity levels, time differences and intensity differences. Brain Res 17:387-405. CrossRef Medline

Bourk TR (1976) Electrical responses of neural units in the anteroventral cochlear nucleus of the cat. PhD thesis, Massachusetts Institute of Technology.

Brand A, Behrend O, Marquardt T, McAlpine D, Grothe B (2002) Precise inhibition is essential for microsecond interaural time difference coding. Nature 417:543-547. CrossRef Medline

Brunso-Bechtold JK, Thompson GC, Masterton RB (1981) HRP study of the organization of auditory afferents ascending to central nucleus of inferior colliculus in cat. J Comp Neurol 197:705-722. CrossRef Medline

Burkard R (2006) Calibration of acoustic transients. Brain Res 1091:27-31. CrossRef Medline

Caird D, Klinke R (1987) Processing of interaural time and intensity differences in the cat inferior colliculus. Exp Brain Res 68:379-392. Medline

Carney LH, Yin TC (1989) Responses of low-frequency cells in the inferior colliculus to interaural time differences of clicks: excitatory and inhibitory components. J Neurophysiol 62:144-161. CrossRef Medline

Carney LH, Yin TC (1988) Temporal coding of resonances by lowfrequency auditory nerve fibers: single-fiber responses and a population model. J Neurophysiol 60:1653-1677. CrossRef Medline

Chung Y, Hancock KE, Delgutte B (2016) Neural coding of interaural time differences with bilateral cochlear implants in unanesthetized rabbits. J Neurosci 36:5520-5531. CrossRef Medline

Day ML, Semple MN (2011) Frequency-dependent interaural delays in the 
medial superior olive: implications for interaural cochlear delays. J Neurophysiol 106:1985-1999. CrossRef Medline

Devore S, Ihlefeld A, Hancock K, Shinn-Cunningham B, Delgutte B (2009) Accurate sound localization in reverberant environments is mediated by robust encoding of spatial cues in the auditory midbrain. Neuron 62:123134. CrossRef Medline

Dynes SB, Delgutte B (1992) Phase-locking of auditory-nerve discharges to sinusoidal electric stimulation of the cochlea. Hear Res 58:79-90. CrossRef Medline

Earl BR, Chertoff ME (2012) Mapping auditory nerve firing density using high-level compound action potentials and high-pass noise masking. J Acoust Soc Am 131:337-352. CrossRef Medline

Fitzpatrick DC, Kuwada S, Batra R (2000) Neural sensitivity to interaural time differences: beyond the Jeffress model. J Neurosci 20:1605-1615. CrossRef Medline

Geisler CD, Rhode WS, Hazelton DW (1969) Responses of inferior colliculus neurons in the cat to binaural acoustic stimuli having wide-band spectra. J Neurophysiol 32:960-974. CrossRef Medline

Glendenning KK, Masterton RB (1983) Acoustic chiasm: efferent projections of the lateral superior olive. J Neurosci 3:1521-1537. CrossRef Medline

Godfrey DA, Kiang NY, Norris BE (1975a) Single unit activity in the posteroventral cochlear nucleus of the cat. J Comp Neurol 162:247-268. CrossRef Medline

Godfrey DA, Kiang NY, Norris BE (1975b) Single unit activity in the dorsal cochlear nucleus of the cat. J Comp Neurol 162:269-284. CrossRef Medline

Goldberg JM, Brown PB (1969) Response of binaural neurons of dog superior olivary complex to dichotic tonal stimuli: some physiological mechanisms of sound localization. J Neurophysiol 32:613-636. CrossRef Medline

Grothe B, Pecka M (2014) The natural history of sound localization in mammals-a story of neuronal inhibition. Front Neural Circuits 8:116. CrossRef Medline

Guinan JJ Jr, Lin T, Cheng H (2005) Medial-olivocochlear-efferent inhibition of the first peak of auditory-nerve responses: evidence for a new motion within the cochlea. J Acoust Soc Am 118:2421-2433. CrossRef Medline

Hamzavi J, Arnoldner C (2006) Effect of deep insertion of the cochlear im plant electrode array on pitch estimation and speech perception. Acta Otolaryngol 126:1182-1187. CrossRef Medline

Hancock KE, Delgutte B (2004) A physiologically based model of interaural time difference discrimination. J Neurosci 24:7110-7117. CrossRef Medline

Hancock KE, Noel V, Ryugo DK, Delgutte B (2010) Neural coding of interaural time differences with bilateral cochlear implants: effects of congenital deafness. J Neurosci 30:14068-14079. CrossRef Medline

Hancock KE, Chung Y, Delgutte B (2013) Congenital and prolonged adultonset deafness cause distinct degradations in neural ITD coding with bilateral cochlear implants. J Assoc Res Otolaryngol 14:393-411. CrossRef Medline

Hartmann R, Topp G, Klinke R (1984) Discharge patterns of cat primary auditory fibers with electrical stimulation of the cochlea. Hear Res 13:4762. CrossRef Medline

He S, Brown CJ, Abbas PJ (2012) Preliminary results of the relationship between the binaural interaction component of the electrically evoked auditory brainstem response and interaural pitch comparisons in bilateral cochlear implant recipients. Ear Hear 33:57-68. CrossRef Medline

Heil P, Peterson AJ (2015) Basic response properties of auditory nerve fibers: a review. Cell Tissue Res 361:129-158. CrossRef Medline

Hu H, Dietz M (2015) Comparison of interaural electrode pairing methods for bilateral cochlear implants. Trends Hear 19: pii: 2331216515617143. CrossRef Medline

Hutson KA, Graña GD, Fitzpatrick DC (2018) Anatomical basis for parallel and interconnected non-ITD pathways from the inferior colliculus to the medial geniculate in the Mongolian gerbil. Assoc Res Otolaryngol 41:28.

Javel E, Shepherd RK (2000) Electrical stimulation of the auditory nerve. III. Response initiation sites and temporal fine structure. Hear Res 140:45-76. CrossRef Medline

Jeffress LA (1948) A place theory of sound localization. J Comp Physiol Psychol 41:35-39. CrossRef Medline

Jercog PE, Svirskis G, Kotak VC, Sanes DH, Rinzel J (2010) Asymmetric excitatory synaptic dynamics underlie interaural time difference processing in the auditory system. PLoS Biol 8:e1000406. CrossRef Medline

Joris PX, Yin TC (1998) Envelope coding in the lateral superior olive. III. comparison with afferent pathways. J Neurophysiol 79:253-269. CrossRef Medline

Joris PX, Van de Sande B, Louage DH, van der Heijden M (2006) Binaural and cochlear disparities. Proc Natl Acad Sci U S A 103:12917-12922. CrossRef Medline

Joshi SN, Dau T, Epp B (2017) A model of electrically stimulated auditory nerve fiber responses with peripheral and central sites of spike generation. J Assoc Res Otolaryngol 18:323-342. CrossRef Medline

Kan A, Litovsky RY (2015) Binaural hearing with electrical stimulation. Hear Res 322:127-137. CrossRef Medline

Kan A, Stoelb C, Litovsky RY, Goupell MJ (2013) Effect of mismatched place-of-stimulation on binaural fusion and lateralization in bilateral cochlear-implant users. J Acoust Soc Am 134:2923-2936. CrossRef Medline

Kiang NYS (1975) Stimulus representation in the discharge patterns of auditory neurons. In: The nervous system: human communication and its disorders, Vol 3 (Tower DB, ed), pp 81-96. New York, NY: Raven.

Kiang NYS, Watanabe T, Thomas EC, Clark LF (1965) Discharge patterns of single fibers in the cat's auditory nerve. MIT ResMonogr 65. Cambridge, MA: MIT.

Kiang NYS, Morest DK, Godfrey DA, Guinan JJ JR, Kane EC (1973) Stimulus coding at caudal levels of the cat's auditory nervous system. I. Response characteristics of single units. In: Basic mechanisms in hearing (Moller AR, ed), pp 455-478. New York, NY: Academic.

Krumbholz K, Patterson RD, Pressnitzer D (2000) The lower limit of pitch as determined by rate discrimination. J Acoust Soc Am 108:1170-1180. CrossRef Medline

Kuwada S, Stanford TR, Batra R (1987) Interaural phase-sensitive units in the inferior colliculus of the unanesthetized rabbit: effects of changing frequency. J Neurophysiol 57:1338-1360. CrossRef Medline

Kuwada S, Fitzpatrick DC, Batra R, Ostapoff EM (2006) Sensitivity to interaural time differences in the dorsal nucleus of the lateral lemniscus of the unanesthetized rabbit: comparison with other structures. J Neurophysiol 95:1309-1322. CrossRef Medline

Laback B, Egger K, Majdak P (2015) Perception and coding of interaural time differences with bilateral cochlear implants. Hear Res 322:138-150. CrossRef Medline

Landsberger DM, Mertens G, Punte AK, Van De Heyning P (2014) Perceptual changes in place of stimulation with long cochlear implant electrode arrays. J Acoust Soc Am 135:EL75-EL81. CrossRef Medline

Landsberger DM, Vermeire K, Claes A, Van Rompaey V, Van de Heyning P (2016) Qualities of single electrode stimulation as a function of rate and place of stimulation with a cochlear implant. Ear Hear 37:e149-159. CrossRef Medline

Leake PA, Snyder RL, Hradek GT, Rebscher SJ (1995) Consequences of chronic extracochlear electrical stimulation in neonatally deafened cats. Hear Res 82:65-80. CrossRef Medline

Litovsky RY, Jones GL, Agrawal S, van Hoesel R (2010) Effect of age at onset of deafness on binaural sensitivity in electric hearing in humans. J Acoust Soc Am 127:400-414. CrossRef Medline

Litovsky R, Parkinson A, Arcaroli J, Sammeth C (2006) Simultaneous bilateral cochlear implantation in adults: a multicenter clinical study. Ear Hear 27:714-731. CrossRef Medline

Lusted HS, Simmons FB (1984) Interaction of cortical evoked potentials to electric and acoustic stimuli. J Acoust Soc Am 76:449-455. CrossRef Medline

Macherey O, Deeks JM, Carlyon RP (2011) Extending the limits of place and temporal pitch perception in cochlear implant users. J Assoc Res Otolaryngol 12:233-251. CrossRef Medline

Macpherson EA, Middlebrooks JC (2002) Listener weighting of cues for lateral angle: the duplex theory of sound localization revisited. J Acoust Soc Am 111:2219-2236. CrossRef Medline

Majdak P, Laback B, Baumgartner WD (2006) Effects of interaural time differences in fine structure and envelope on lateral discrimination in electric hearing. J Acoust Soc Am 120:2190-2201. CrossRef Medline

Maki K, Furukawa S, Hirahara T (2003) Acoustical cues for sound localization by gerbils in an ecological realistic environment. Assoc Res Otolaryngol 26:89.

Mardia KV (1972) Statistics of directional data. London, UK: Academic. 
McAlpine D, Grothe B (2003) Sound localization and delay lines-Do mammals fit the model? Trends Neurosci 26:347-350. CrossRef Medline

McAlpine D, Jiang D, Shackleton TM, Palmer AR (1998) Convergent input from brainstem coincidence detectors onto delay-sensitive neurons in the inferior colliculus. J Neurosci 18:6026-6039. CrossRef Medline

McAlpine D, Jiang D, Palmer AR (2001) A neural code for low-frequency sound localization in mammals. Nat Neurosci 4:396-401. CrossRef Medline

McKay CM, Carlyon RP (1999) Dual temporal pitch percepts from acoustic and electric amplitude-modulated pulse trains. J Acoust Soc Am 105:347357. CrossRef Medline

Middlebrooks JC, Snyder RL (2010) Selective electrical stimulation of the auditory nerve activates a pathways specialized for high temporal acuity. J Neurosci 30:1937-1946. CrossRef Medline

Miller CA, Abbas PJ, Robinson BK, Rubinstein JT, Matsuoka AJ (1999) Electrically evoked single-fiber action potentials from cat: responses to monopolar, monophasic stimulation. Hear Res 130:197-218. CrossRef Medline

Miller CA, Abbas PJ, Robinson BK, Nourski KV, Zhang F, Jeng FC (2006) Electrical excitation of the acoustically sensitive auditory nerve: singlefiber responses to electric pulse trains. J Assoc Res Otolaryngol 7:195-210. CrossRef Medline

Moxon EC (1971) Neural and mechanical responses to electric stimulation of the cat's inner ear. $\mathrm{PhD}$ thesis, Massachusetts Institute of Technology.

Pecka M, Brand A, Behrend O, Grothe B (2008) Interaural time difference processing in the mammalian medial superior olive: the role of glycinergic inhibition. J Neurosci 28:6914-6925. CrossRef Medline

Pfeiffer RR (1966) Classification of response patterns of spike discharges for units in the cochlear nucleus: tone-burst stimulation. Exp Brain Res 1:220-235. Medline

Pfeiffer RR, Kim DO (1972) Response patterns of single cochlear nerve fibers to clock stimuli: descriptions for cat. J Acoust Soc Am 52:1669-1677. CrossRef Medline

Plauška A, van der Heijden M, Borst JGG (2017) A test of the stereausis hypothesis for sound localization in mammals. J Neurosci 37:7278-7289. CrossRef Medline

Ricketts TA, Grantham DW, Ashmead DH, Haynes DS, Labadie RF (2006) Speech recognition for unilateral and bilateral cochlear implant modes in the presence of uncorrelated noise sources. Ear Hear 27:763-773. CrossRef Medline

Sakitt B (1973) Indices of discriminability. Nature 241:133-134. CrossRef Medline

Sato M, Baumhoff P, Kral A (2016) Cochlear implant stimulation of a hearing ear generates separate electrophonic and electroneural responses. J Neurosci 36:54-64. CrossRef Medline

Schatzer R, Vermeire K, Visser D, Krenmayr A, Kals M, Voormolen M, Van de Heyning P, Zierhofer C (2014) Electric-acoustic pitch comparisons in single-sided-deaf cochlear implant users: frequency-place functions and rate pitch. Hear Res 309:26-35. CrossRef Medline

Seeber BU, Fastl H (2008) Localization cues with bilateral cochlear implants. J Acoust Soc Am 123:1030-1042. CrossRef Medline

Seidl AH, Grothe B (2005) Development of sound localization mechanisms in the mongolian gerbil is shaped by early acoustic experience. J Neurophysiol 94:1028-1036. CrossRef Medline

Shamma SA, Shen NM, Gopalaswamy P (1989) Stereausis: binaural processing without neural delays. J Acoust Soc Am 86:989-1006. CrossRef Medline

Shepherd RK, Javel E (1999) Electrical stimulation of the auditory nerve: II. effect of stimulus waveshape on single fibre response properties. Hear Res 130:171-188. CrossRef Medline
Siveke I, Pecka M, Seidl AH, Baudoux S, Grothe B (2006) Binaural response properties of low-frequency neurons in the gerbil dorsal nucleus of the lateral lemniscus. J Neurophysiol 96:1425-1440. CrossRef Medline

Smith ZM, Delgutte B (2007) Sensitivity to interaural time differences in the inferior colliculus with bilateral cochlear implants. J Neurosci 27:67406750. CrossRef Medline

Spitzer MW, Semple MN (1995) Neurons sensitive to interaural phase disparity in gerbil superior olive: diverse monaural and temporal response properties. J Neurophysiol 73:1668-1690. CrossRef Medline

Stahl P, Macherey O, Meunier S, Roman S (2016) Rate discrimination at low pulse rates in normal-hearing and cochlear implant listeners: Influence of intracochlear stimulation site. J Acoust Soc Am 139: 1578-1591. CrossRef Medline

Stange A, Myoga MH, Lingner A, Ford MC, Alexandrova O, Felmy F, Pecka M, Siveke I, Grothe B (2013) Adaptation in sound localization: from GABA(B) receptor-mediated synaptic modulation to perception. Nat Neurosci 16:1840-1847. CrossRef Medline

Stecker GC, Harrington IA, Middlebrooks JC (2005) Location coding by opponent neural populations in the auditory cortex. PLoS Biol 3:e78. CrossRef Medline

Takesian AE, Kotak VC, Sanes DH (2009) Developmental hearing loss disrupts synaptic inhibition: implications for auditory processing. Future Neurol 4:331-349. CrossRef Medline

Tillein J, Hubka P, Syed E, Hartmann R, Engel AK, Kral A (2010) Cortical representation of interaural time difference in congenital deafness. Cereb Cortex 20:492-506. CrossRef Medline

Undurraga JA, Haywood NR, Marquardt T, McAlpine D (2016) Neural representation of interaural time differences in humans-an objective measure that matches behavioural performance. J Assoc Res Otolaryngol 17: 591-607. CrossRef Medline

van den Honert C, Stypulkowski PH (1984) Physiological properties of the electrically stimulated auditory nerve. II. Single fiber recordings. Hear Res 14:225-243. CrossRef Medline

van Hoesel RJ (2012) Contrasting benefits from contralateral implants and hearing aids in cochlear implant users. Hear Res 288:100-113. CrossRef Medline

Vollmer M, Bonham BH, Tillein J (2008) Spectral and temporal interactions in cat inferior colliculus of combined electric and acoustic stimulation of the hearing cochlea. Assoc Res Otolaryngol 31:298.

Wiegner A, Wright CG, Vollmer M (2016) Multichannel cochlear implant for selective neuronal activation and chronic use in the free-moving mongolian gerbil. J Neurosci Methods 273:40-54. CrossRef Medline

Wightman FL, Kistler DJ (1992) The dominant role of low-frequency interaural time differences in sound localization. J Acoust Soc Am 91:16481661. CrossRef Medline

Yin TC, Chan JC (1990) Interaural time sensitivity in medial superior olive of cat. J Neurophysiol 64:465-488. CrossRef Medline

Yin TC, Kuwada S (1983a) Binaural interaction in low-frequency neurons in inferior colliculus of the cat. II. effects of changing rate and direction of interaural phase. J Neurophysiol 50:1000-1019. CrossRef Medline

Yin TC, Kuwada S (1983b) Binaural interaction in low-frequency neurons in inferior colliculus of the cat. III. Effects of changing frequency. J Neurophysiol 50:1020-1042. CrossRef Medline

Yin TC, Chan JC, Carney LH (1987) Effects of interaural time delays of noise stimuli on low-frequency cells in the cat's inferior colliculus. III. Evidence for cross-correlation. J Neurophysiol 58:562-583. CrossRef Medline

Yin TC, Chan JC, Irvine DR (1986) Effects of interaural time delays of noise stimuli on low-frequency cells in the cat's inferior colliculus. I. Responses to wideband noise. J Neurophysiol 55:280-300. CrossRef Medline 